\title{
Electromagnetic Processes in Doubly-Nonlinear Composites
}

\author{
A. VISINTIN \\ Dipartimento di Matematica, Università degli Studi di Trento, \\ Trento, Italy
}

Electromagnetic processes in inhomogeneous conductors are here described by coupling the Maxwell equations with nonlinear constitutive relations of the form $\vec{B}=\vec{B}(\vec{H}, x)$ and $\vec{J}=\vec{J}(\vec{E}, \vec{H}, x)$, neglecting hysteresis and displacement currents. The latter equality may also account for the Hall effect.

A doubly-nonlinear parabolic-hyperbolic equation is formulated, and existence of a solution is proved via approximation by time-discretization, derivation of a priori estimates, and passage to the limit via compensated compactness and compactness by strict convexity.

It is then assumed that the medium is a composite that exhibits periodic oscillations in space. Convergence to a corresponding homogenized two-scale problem is proved as the oscillation period vanishes, via Nguetseng's notion of twoscale convergence. Finally this two-scale formulation is proved to be equivalent to a coarse-scale problem.

Keywords Electromagnetism; Homogenization; Maxwell equations; Two-scale convergence.

Mathematics Subject Classification 35K60; 35R35; 78A25; 78M40.

\section{Introduction}

In this paper we deal with electromagnetic processes in a composite conductor, e.g., the mixture of two metals, under an applied electromotive force; for instance this may model an electric transformer. We neglect hysteresis and represent the constitutive behaviour by means of two nonlinear relations of the form

$$
\vec{B}=\vec{B}(\vec{H}, x), \quad \vec{J}=\vec{J}(\vec{E}, \vec{H}, x) .
$$

The Physical Problem. The first condition may represent the magnetic behaviour of several metals, including soft iron and mild steel; the second one is a nonlinear

Received December 1, 2006; Accepted September 1, 2007

Address correspondence to A. Visintin, Dipartimento di Matematica, Università degli Studi di Trento, via Sommarive 14, 38050 Povo (Trento), Italy; E-mail: Visintin@ science.unitn.it 
conduction law, and may account for dependence of the electric conductivity on the magnetic field $\vec{H}$, as it is prescribed by the classical Hall effect. We assume that the source field does not vary too rapidly in time, so that we may neglect displacement currents by the so-called eddy-current approximation. In this case the Maxwell system yields a quasi-linear parabolic system of P.D.E.s.

If the body is surrounded by an insulating medium like air, there displacement currents must be taken into account. This yields a linear hyperbolic system outside the domain $\Omega$ occupied by the body, that must be coupled with the parabolic problem in $\Omega$. We thus formulate our problem in the whole space: due to the properties of electromagnetic radiation, it would not be natural to confine the Maxwell system to $\Omega$ and to prescribe conditions on the boundary (Bossavit, 1993; Bossavit and Verité, 1981).

We provide an initial-value problem for the following system of equations. Let us denote by $\chi_{\Omega}$ the characteristic function of $\Omega$, and set $\Omega^{\prime}:=\mathbf{R}^{3} \backslash \Omega$. We search for four fields $\vec{B}, \vec{H}, \vec{E}, \vec{J}$ such that, omitting constant coefficients and denoting by $\chi_{\Omega}$ the characteristic function of $\Omega$,

$$
\begin{gathered}
\left.\nabla \times \vec{H}=\vec{J}+\left(1-\chi_{\Omega}\right) \frac{\partial \vec{E}}{\partial t}+\vec{g} \quad \text { in } \mathbf{R}^{3} \times\right] 0, T[\quad(\nabla \times:=\text { curl }), \\
\left.\nabla \times \vec{E}=-\frac{\partial \vec{B}}{\partial t} \text { in } \mathbf{R}^{3} \times\right] 0, T[ \\
\vec{B} \in \partial \varphi(\vec{H}, x) \quad \text { in } \Omega \times] 0, T\left[, \quad \vec{B}=\vec{H} \quad \text { in } \Omega^{\prime} \times\right] 0, T[, \\
\vec{J}=\vec{\alpha}(\vec{E}, \vec{H}, x) \quad \text { in } \Omega \times] 0, T\left[, \quad \vec{J}=\overrightarrow{0} \quad \text { in } \Omega^{\prime} \times\right] 0, T[, \\
\vec{E}(\cdot, 0)=\vec{E}^{0} \quad \text { in } \Omega, \quad \vec{B}(\cdot, 0)=\vec{B}^{0} \quad \text { in } \mathbf{R}^{3} .
\end{gathered}
$$

Here $\vec{g}, \vec{E}^{0}$ and $\vec{B}^{0}$ are prescribed fields, with $\vec{g}$ and $\vec{B}^{0}$ divergence-free. We assume that the function $\varphi(\cdot, x)$ is strictly convex and lower semicontinuous, and denote its subdifferential by $\partial \varphi$ (e.g., Ekeland and Temam, 1974; Hiriart-Urruty and Lemarechal, 1993; Rockafellar, 1969). We do not exclude discontinuities in the constitutive relation between $\vec{B}$ and $\vec{H}$; they may correspond to the occurrence of unknown moving interfaces, i.e., free boundaries. On the other hand we assume that the function $\vec{\alpha}(\vec{E}, \vec{H}, x)$ is monotone nondecreasing w.r.t. $\vec{E}$ and continuous w.r.t. the pair $(\vec{E}, \vec{H})$. (The continuity w.r.t. $\vec{E}$ is suggested by mathematical convenience, whereas that w.r.t. $\vec{H}$ seems necessary for the analysis.) The relation $\vec{J}=\vec{\alpha}(\vec{E}, \vec{H}, x)$ obviously includes the Ohm law

$$
\vec{J}=\sigma\left(\vec{E}+\vec{E}_{a}\right)
$$

here $\sigma$ is either a positive scalar or (more generally) a positive-definite $3 \times 3$-tensor, and represents the electric conductivity; $\vec{E}_{a}$ is a prescribed applied electromotive force. In Sec. 2 we shall see that the Hall effect may be represented by the equation

$$
\left.\vec{J}=\sigma\left(\vec{E}+h \vec{J} \times \vec{B}+\vec{E}_{a}\right) \text { in } \Omega \times\right] 0, T[,
$$

$h$ being a physical constant, and that if $\partial \varphi$ is single-valued this relation may be reduced to the form (1.5). This conduction law thus allows for a certain generality. The energy integral that we shall derive afterwards will also show that this model is 
consistent with the second principle of thermodynamics, whenever the mapping $\vec{\alpha}$ is nondecreasing w.r.t. its first argument.

Existence of a Solution. If the relation between $\vec{J}$ and $\vec{E}$ were linear and independent of $\vec{H}$, of the form $\vec{E}=A(x) \cdot \vec{J}$ say, then it would be possible to eliminate the fields $\vec{E}$ and $\vec{J}$ in $\Omega$, and thus get

$$
\left\{\begin{array}{l}
\frac{\partial \vec{B}}{\partial t}+\nabla \times[A(x) \cdot \nabla \times \vec{H}]=\nabla \times[A(x) \cdot \vec{g}] \\
\vec{B} \in \partial \varphi(\vec{H}, x)
\end{array} \quad \text { in } \Omega \times\right] 0, T[.
$$

This would fit the classical theory of first-order evolution equations governed by maximal monotone operators, and the problem would then be well-posed. On the other hand the occurrence of a double nonlinearity makes the system (1.2)-(1.6) nontrivial; the noncompactness of the injection $L_{\text {rot }}^{2}(\Omega)^{3} \rightarrow L^{2}(\Omega)^{3}$ appears as a further element of difficulty.

Here we provide a weak formulation in Sobolev spaces for an initial-value problem associated to the system (1.2)-(1.6), and prove existence of a solution, via approximation by time-discretization, derivation of a priori estimates, and passage to the limit. For the latter step we use Murat's and Tartar's theory of compensated compactness in the form of the div-curl lemma; we then apply compactness by strict convexity as well as more standard techniques.

Two- and Single-Scale Homogenization. The main purpose of this paper is to study processes in a composite material, and to derive a homogenized model; namely to devise the constitutive equations of an effective fictitious material, whose macroscopic electromagnetic behaviour should be undistinguishable from that of the composite.

By a well-known procedure, we represent the inhomogeneous behaviour by assuming that the functions $\varphi$ and $\vec{\alpha}$ depend not only on $x$ but also on $x / \varepsilon$ ( $\varepsilon$ being a small scalar parameter), and that the latter dependence is periodic. We then let $\varepsilon$ vanish, and show two-scale convergence (in the sense of Nguetseng) to a solution of a two-scale problem; this comprises two systems, that are respectively set in terms of coarse- and fine-scale fields and are coupled by the constitutive relations. Our argument rests on the two-scale extension of the compactness results that are at the basis of the above existence theorem, namely, compensated compactness and compactness by strict convexity.

The formulation of a two-scale model raises the question of upscaling, namely of deriving a purely coarse-scale model by eliminating any dependence on the finescale variable $y$. We thus retrieve a coarse-scale problem of the form (1.2)-(1.6), with different constitutive functions $\varphi$ and $\vec{\alpha}$. These functions are determined via the solution of two families of nonlinear cell problems; for them we also construct a solution in the case of the Ohm law. One might thus claim that the problems of the form (1.2)-(1.6) are stable by homogenization.

We also show that, conversely, any solution of the coarse-scale problem is the fine-scale average of some solution of the two-scale problem. These two formulations are thus equivalent, and by dealing with the coarse-scale model there is no risk of introducing nonphysical spurious solutions. This also entails that one cannot distinguish a composite from a mesoscopically homogeneous material just on 
the basis of the macroscopic behaviour. This part is based on techniques of Visintin (2007b), and is one of the main issues of this paper.

Analogous developments apply to similar equations that model different phenomena. For instance, a doubly-nonlinear parabolic equation arises as a model of thermal evolution in presence of phase transitions: besides the nonlinear relation between the temperature and the density of internal energy, one may assume a generalized Fourier's conduction law, that prescribes the heat flux as a nonlinear function of the temperature and of the temperature gradient. By coupling these constitutive relations with the energy balance equation, one gets a generalization of the classical Stefan model; the homogenization of this problem is studied by Visintin (2007c).

Literature. Homogenization has a well-established tradition, see e.g., the works of Allaire (2002), Attouch (1984), Bakhvalov and Panasenko (1989), Bensoussan et al. (1978), Braides and Defranceschi (1998), Cioranescu and Donato (1999), Dal Maso (1993), De Giorgi and Spagnolo (1973), Jikov et al. (1994), Marcellini (1978), Milton (2002), Murat and Tartar (1997), Sanchez-Palencia (1980), and Tartar (1977); in particular the homogenization of the Maxwell system was addressed e.g., by Bensoussan et al. (1978), Jikov et al. (1994), and SanchezPalencia (1980). The homogenization of integral functionals was studied by Carbone and Sbordone (1979), Marcellini (1978), see also Braides and Defranceschi (1998), Carbone and De Arcangelis (2001), Cioranescu et al. (2004, 2006), and Dal Maso (1993).

Two-scale convergence was introduced by Nguetseng (1989) and then developed by Allaire (1992); see also e.g., Arbogast et al. (1990), Bourgeat et al. (1996), Cioranescu et al. (2002), and Lukkassen and Nguetseng (2002) for a recent review. This notion is now applied in a growing number of papers; for instance in Birnir and Wellander (2006), Wellander (2002), and Wellander and Kristensson (2003) it was used for the homogenization of linear problems based on the Maxwell system.

The theory of compensated compactness was developed by Murat (1978, 1981, 1987), Murat and Tartar (1997), and Tartar (1977, 1978, 1979, 2002). Compactness by strict convexity was introduced by Visintin (1984), cf. also Visintin (1996, Chap. X). The analysis of the Maxwell equations requires the use of some variants of the classical Sobolev spaces, that are studied e.g., by Cessenat (1996) and Dautray and Lions (1988). The homogenization of the Maxwell equations coupled with a single nonlinear constitutive relation was studied in Bossavit and Damlamian (1981), Codegone and Negro (1982, 1984), Damlamian (1981), and Negro (1987). Twoscale convergence was also applied to the homogenization of electric circuits e.g., by Lenczner (1997) and Lenczner and Senouci (1999).

Several works were devoted to doubly-nonlinear parabolic equations, see e.g., Alt and Luckhaus (1983), Arai (1979), Blanchard and Francfort (1988, 1991), Blanchard and Porretta (2005), Carroll and Showalter (1976), Colli (1992), Colli and Visintin (1990), DiBenedetto and Showalter (1981), Grange and Mignot (1972), Lions (1969), and Visintin (1996). These equations arise as models of a number of phenomena, including phase transitions and filtration of either gas or liquid through porous media, see e.g., Alt and Luckhaus (1983); their homogenization was studied for instance by Bourgeat et al. (1996), Hornung (1997), and Nandakumaran and Rajesh (2001). In the latter work oscillations w.r.t. the time-variable were also accounted for in the elliptic term, and space-time two-scale convergence was used. 
Uniqueness of the solution was proved by Blanchard and Porretta (2005), Carrillo (1999), Carrillo and Wittbold (1999), and Igbida and Urbano (2003) via the notions of entropy solutions, renormalization, and $L^{1}$-contractions. The latter results apply to a wide class of problems, that however does not seem to include that addressed in the present work.

In Duvaut and Lions (1972) the break-down of an aerial under an overcritical electric field was formulated and studied as an obstacle problem for the Ohm law. This also may be represented as a nonlinear conduction law of the form (1.5), in which $\vec{J}$ is an unbounded function of $\vec{E}$. In this framework the coupling with the nonlinear relation (1.4) does not seem obvious. In Birnir and Wellander (2006) a model for ceramic varistors was formulated by coupling the Maxwell equations with a nonlinear conduction law; the homogenization was then studied via two-scale convergence.

The Hall effect is illustrated in many physical texts, see e.g., Ashcroft and Mermin (1976), Jones (1964), and Landau and Lifshitz (1960), but so far the coupled system of the Maxwell and Hall equations seems to have raised little concern among mathematical analysts, although the nondissipative character of the latter might attract attention. In Visintin (1985) this effect was accounted for by writing the electric conduction law in the form $\vec{J}=k(\vec{H}) \nabla \times \vec{H}$, and existence of a weak solution was proved. That result rests on a fixed-point argument that does not seem to take over to the present more general setting. This author knows of no other paper dealing with the analysis of the Hall law in Sobolev spaces.

This work is in the framework of a research about two-scale convergence that also lead to study applications to thermal diffusion (Visintin, 2007c) and continuum mechanics (Visintin, 2006b). Some of the present results are based on the works (Visintin, 2007a,b, in preparation) and were announced in Visintin (2006a).

Plan of the Paper. In Sec. 2 we illustrate the electromagnetic model and derive the system (1.2)-(1.6). The next two sections are preparatory; their content is not original, but is needed in the remainder of this article. In Sec. 3 we review the notion of two-scale convergence along the lines of the seminal works (Allaire, 1992; Nguetseng, 1989); we also state results about the two-scale limit of the curl and divergence operators, cf. Visintin (2007a), and a simple two-scale extension of the div-curl lemma. In Sec. 4 we outline some variational properties of two-scale convergence, including a two-scale extension of the theorem of compactness by strict convexity, cf. Visintin (2007b).

In Sec. 5 we provide a weak formulation of an initial-value problem for our parabolic-hyperbolic system, and show existence of a weak solution. Our argument is based on approximation by time-discretization and on classical results of the theory of maximal monotone operators, cf. e.g., Barbu (1976), Brezis (1973), Browder (1970), and Lions (1969). In Sec. 6 we pass to the limit as the space-period $\varepsilon$ vanishes and prove convergence to a solution of a two-scale homogenized problem via the results of Secs. 3 and 4. In Sec. 7 we complete the homogenization procedure by showing that the fine-scale average of the solution of the two-scale formulation solves a single-scale problem, and that conversely any solution of the latter problem can be retrieved by upscaling. We also display the calculation of the homogenized conductivity tensor in the case of the Ohm law. Finally in Sec. 8 we discuss some further questions and draw our conclusions. 


\section{Maxwell's Equations and Constitutive Laws}

Maxwell's System. Let us consider a conductor that occupies a (possibly unbounded and multiply-connected) domain $\Omega$ of $\mathbf{R}^{3}$, fix a constant $T>0$, and set $\left.A_{t}:=A \times\right] 0, t[$ for any $A \subset \mathbf{R}^{3}$ and any $\left.t \in\right] 0, T[$. With standard notation we denote the magnetic field by $\vec{H}$, the magnetic induction by $\vec{B}$, the electric field by $\vec{E}$, the electric displacement by $\vec{D}$, the electric current density by $\vec{J}$, the speed of light in vacuum by $c$, the dielectric constant by $\epsilon$, and the electric charge density by $\rho_{\mathrm{el}}$. In Gauss units the full system of Maxwell's equations reads

$$
\begin{gathered}
c \nabla \times \vec{H}=4 \pi \vec{J}+\frac{\partial \vec{D}}{\partial t} \quad \text { in } \mathbf{R}_{T}^{3}(\nabla \times:=\text { curl }), \\
c \nabla \times \vec{E}=-\frac{\partial \vec{B}}{\partial t} \quad \text { in } \mathbf{R}_{T}^{3}, \\
\nabla \cdot \vec{B}=0 \quad \text { in } \mathbf{R}_{T}^{3}(\nabla \cdot:=\mathrm{div}), \\
\nabla \cdot \vec{D}=4 \pi \rho_{\mathrm{el}} \quad \text { in } \mathbf{R}_{T}^{3} .
\end{gathered}
$$

By (2.1) and by the continuity equation $\nabla \cdot \vec{J}+\partial \rho_{\mathrm{el}} / \partial t=0$, if $\partial \rho_{\mathrm{el}} / \partial t=0$ in $\mathbf{R}_{T}^{3}$ then $\partial \nabla \cdot \vec{D} / \partial t=\overrightarrow{0}$. Similarly $\partial \nabla \cdot \vec{B} / \partial t=\overrightarrow{0}$ by (2.2). If we prescribe the Gauss equations (2.3) and (2.4) at $t=0$ we then retrieve them for any instant.

We assume that the fields do not vary too rapidly in time, and accordingly neglect displacement currents, $\partial \vec{D} / \partial t$, in the conductor (so-called eddy current approximation). We also assume that $\vec{J}$ equals a prescribed time-dependent field, $\vec{g}$, outside $\Omega$, and set $\vec{g}:=\overrightarrow{0}$ in $\Omega$. As $\vec{D}=\epsilon \vec{E}$ outside $\Omega$, (2.1) then reads

$$
c \nabla \times \vec{H}=4 \pi \chi_{\Omega} \vec{J}+4 \pi \vec{g}+\epsilon\left(1-\chi_{\Omega}\right) \frac{\partial \vec{E}}{\partial t} \text { in } \mathbf{R}_{T}^{3}
$$

The above equations will be coupled with initial conditions for $\vec{B}$ in $\mathbf{R}^{3}$ and for $\vec{E}$ in $\Omega^{\prime}$, with suitable restrictions on the behaviour at infinity, and with appropriate constitutive relations. Physically it would be less natural to confine the Maxwell system to $\Omega$ and then to prescribe conditions on its boundary, cf. Bossavit (1993) and Bossavit and Verité (1981).

In order to make formulas more readable, henceforth we omit all physical constants, as well as the factor $4 \pi$ in (2.1) and (2.5). This abuse of notation is justified for our analysis can be extended to the equations with the actual physical coefficients without any difficulty. For instance (2.5) thus reads

$$
\nabla \times \vec{H}=\chi_{\Omega} \vec{J}+\vec{g}+\left(1-\chi_{\Omega}\right) \frac{\partial \vec{E}}{\partial t} \quad \text { in } \mathbf{R}_{T}^{3} .
$$

Constitutive Relations. We assume a relation between $\vec{B}$ and $\vec{H}$ of the form

$$
\vec{B}(x, t) \in \partial \varphi(\vec{H}(x, t), x) \text { for a.e. }(x, t) \in \mathbf{R}_{T}^{3} \text {. }
$$

By the definition of subdifferential, this inclusion means that $\vec{H}(x, t)$ minimizes the functional $J(\vec{v}):=\int_{\mathbf{R}^{3}}[\varphi(\vec{v}(x), x)-\vec{B}(x, t) \cdot \vec{v}(x)] d x$. This relation may model the nonlinear behaviour of a large class of materials, including soft iron and mild 
steel, in which hysteresis effects may be neglected. Our hypotheses will not exclude discontinuities in the $\vec{B}$ vs. $\vec{H}$ constitutive relation; these may correspond to the occurrence of unknown moving interfaces, also called free boundaries, across which the fields $\vec{B}$ and $\vec{E}$ fulfil discontinuity relations of Rankine-Hugoniot-type; cf. e.g., Visintin (1996, Sec. IV.8).

Let us now come to the conduction law. Denoting a prescribed applied electromotive force by $\vec{E}_{a}$ and the electric conductivity-tensor by $\sigma$, the Ohm law reads

$$
\vec{J}=\sigma \cdot\left(\vec{E}+\vec{E}_{a}\right) \text { in } \Omega_{T}
$$

In this paper we deal with a more general relation of the form

$$
\vec{J}=\vec{\alpha}(\vec{E}, \vec{H}, x) \text { in } \Omega_{T} .
$$

The Maxwell equations (2.1), (2.2) coupled with the constitutive laws (2.7), (2.9) constitute a doubly-nonlinear parabolic equation in $\Omega_{T}$ and a linear hyperbolic equation in $\Omega_{T}^{\prime}$.

The Hall Law. The relation (2.9) allows for the dependence of the electric conductivity on the magnetic field $\vec{H}$. This may be compared with the Hall law, that accounts for the onset of an electromotive force in presence of an electric current and of a magnetic induction field $\vec{B}$ :

$$
\vec{E}_{h}=h \vec{J} \times \vec{B} \quad(h \text { being a physical constant })
$$

see e.g., Ashcroft and Mermin (1976, pp. 11-15), Jones (1964, p. 737), Landau and Lifshitz (1960, Sec. 21). This behaviour is due to the Lorentz force

$$
\vec{F}=\rho E+c^{-1} \rho \vec{v} \times \vec{B} \quad(c \text { being the speed of light }),
$$

that acts on any particle of electric charge $\rho$ in motion with speed $\vec{v}$ in a field $\vec{B}$. This explains why $\vec{B}$ rather than $\vec{H}$ occurs in (2.10). ${ }^{1}$ In this case the Ohm law (2.8) is replaced by

$$
\vec{J}=\sigma \cdot\left(\vec{E}+\vec{E}_{h}+\vec{E}_{a}\right)=\sigma \cdot\left(\vec{E}+h \vec{J} \times \vec{B}+\vec{E}_{a}\right) \text { in } \Omega_{T} .
$$

For any $\vec{B}$ and $\vec{E}_{a}$, let $\vec{E}_{i}$ and $\vec{J}_{i}$ be related by this equation for $i=1,2$. Setting $\tilde{\vec{E}}=\vec{E}_{1}-\vec{E}_{2}$ and $\tilde{\vec{J}}=\vec{J}_{1}-\vec{J}_{2}$ we then have

$$
\tilde{\vec{J}}=\sigma \cdot(\tilde{\vec{E}}+h \tilde{\vec{J}} \times \vec{B}), \quad \text { whence }(\sigma \cdot \tilde{\vec{E}}) \cdot \tilde{\vec{J}}=|\tilde{\vec{J}}|^{2}
$$

For any $\vec{B}$, the field $\vec{E}$ is thus a linear and strictly monotone function of $\vec{J}$ and conversely, so that the linear relation (2.11) also reads

$$
\vec{J}=\mathscr{A}(\vec{B}) \cdot\left(\vec{E}+\vec{E}_{a}\right),
$$

${ }^{1}$ Some authors write $\vec{H}$ in place of $\vec{B}$ in (2.10), but then $\vec{B}$ should be assumed to be proportional to $\vec{H}$. 
$\mathscr{A}(\vec{B})$ being an asymmetric positive-definite $3 \times 3$-tensor that linearly depends on $\vec{B}$. If $\partial \varphi$ is single-valued, setting $\tilde{A}:=\mathscr{A} \circ \partial \varphi$ by (2.7) we get

$$
\vec{J}=\tilde{\mathscr{A}}(\vec{H}) \cdot\left(\vec{E}+\vec{E}_{a}\right) \text { in } \Omega_{T},
$$

and this is encompassed by the condition (2.9). If $\partial \varphi$ is continuous then $\tilde{\mathscr{A}}$ is also continuous, consistently with the hypotheses that we shall assume in the next sections. However if $\partial \varphi$ is multivalued this reduction is not possible, and in this case the analysis of the Hall effect is here left as an open question.

\section{Two-Scale Convergence}

In view of the study of homogenization in this section we collect some properties of two-scale convergence, mainly along the lines of the seminal works (Allaire, 1992; Nguetseng, 1989). We also deal with some differential properties and show a simple two-scale extension of the div-curl lemma.

Let us set $Y:=\left[0,1\left[{ }^{3}\right.\right.$, denote by $\mathcal{Y}$ the same set equipped with the metric of the 3-dimensional (flat) unit torus, and identify any $Y$-periodic function on $\mathbf{R}^{3}$ with a function on $\mathcal{Y}$. Let us denote by $\varepsilon$ a parameter that we assume to vanish along a prescribed sequence. Let $p \in[1,+\infty],\left\{u_{\varepsilon}\right\}$ be any bounded sequence of $L^{p}\left(\mathbf{R}^{3}\right)$, and $u \in L^{p}\left(\mathbf{R}^{3} \times \mathscr{Y}\right)$; by an obvious extension of Nguetseng's definition of Nguetseng (1989), we say that $u_{\varepsilon}$ weakly (weakly star, if $p=\infty$ ) two-scale converges to $u$ in $L^{p}\left(\mathbf{R}^{3} \times \mathcal{Y}\right)$, and write $u_{\varepsilon} \underset{2}{\rightarrow} u\left(u_{\varepsilon} \underset{2}{\stackrel{*}{\rightarrow}} u\right.$, if $\left.p=\infty\right)$, whenever

$$
\int_{\mathbf{R}^{3}} u_{\varepsilon}(x) v(x, x / \varepsilon) d x \rightarrow \iint_{\mathbf{R}^{3} \times \mathscr{y}} u(x, y) v(x, y) d x d y \quad \forall v \in \mathscr{D}\left(\mathbf{R}^{3} \times \mathscr{Y}\right) .
$$

If $p \in] 1,+\infty$ [ following Allaire (1992) we say that $u_{\varepsilon}$ strongly two-scale converges to $u$ in $L^{p}\left(\mathbf{R}^{3} \times \mathcal{Y}\right)$, and write $u_{\varepsilon} \underset{2}{\rightarrow} u$, if (3.1) holds and $\left\|u_{\varepsilon}\right\|_{L^{p}\left(\mathbf{R}^{3}\right)} \rightarrow\|u\|_{L^{p}\left(\mathbf{R}^{3} \times y\right)}$. As for time-dependent functions, if $r, s \in] 1,+\infty],\left\{u_{\eta}\right\}$ is a bounded sequence of $L^{s}\left(0, T ; L^{r}\left(\mathbf{R}^{3}\right)\right)$ and $u \in L^{s}\left(0, T ; L^{r}\left(\mathbf{R}^{3} \times \mathcal{Y}\right)\right),{ }^{2}$ we say that $u_{\eta} \underset{2}{\overrightarrow{2}} u\left(u_{\eta} \underset{2}{\stackrel{*}{2}} u\right.$ if either $r=\infty$ or $s=\infty$ ) whenever

$\iint_{\mathbf{R}_{T}^{3}} u_{\eta}(x, t) v(x, x / \eta, t) d x d t \rightarrow \iiint_{\mathbf{R}_{T}^{3} \times \mathscr{Y}} u(x, y, t) v(x, y, t) d x d y d t \quad \forall v \in \mathscr{D}\left(\mathbf{R}_{T}^{3} \times \mathscr{Y}\right)$.

These definitions and the next two statements take over to functions defined on a subdomain of $\mathbf{R}^{3}$, just by extending them with vanishing value outside that domain. We shall denote the standard (single-scale) weak (weak star, strong, resp.) convergence by $\rightarrow(\stackrel{*}{\rightarrow}, \rightarrow$, resp. $)$.

Proposition 3.1 (Allaire, 1992; Nguetseng, 1989). For any bounded sequence $\left\{u_{\varepsilon}\right\}$ of $\left.L^{p}\left(\mathbf{R}^{3}\right)(p \in 1,+\infty]\right)$, there exists $u \in L^{p}\left(\mathbf{R}^{3} \times \mathcal{Y}\right)$ such that, possibly extracting $a$

${ }^{2}$ It is known that for $r=\infty$ difficulties arise in defining these spaces, for $L^{\infty}(\Omega)$ and $L^{\infty}(\Omega \times Y)$ are not separable. This drawback may be removed by assuming that these vector-valued functions are weakly star measurable. 
subsequence,

$$
u_{\varepsilon} \underset{2}{\rightarrow} u \text { in } L^{p}\left(\mathbf{R}^{3} \times \mathcal{Y}\right)\left(u_{\varepsilon} \underset{2}{\stackrel{*}{\longrightarrow}} u \text { if } p=\infty\right)
$$

Proposition 3.2 (Allaire, 1992). If $\left\{u_{\varepsilon}\right\}$ is a sequence of $L^{p}\left(\mathbf{R}^{3}\right)(p \in[1,+\infty[)$ such that $u_{\varepsilon} \underset{2}{\rightarrow} u$ in $L^{p}\left(\mathbf{R}^{3} \times \mathcal{Y}\right)$, then

$$
\begin{gathered}
u_{\varepsilon} \rightarrow \hat{u}:=\int_{y} u(\cdot, y) d y \text { in } L^{p}\left(\mathbf{R}^{3}\right), \\
\liminf _{\varepsilon \rightarrow 0}\left\|u_{\varepsilon}\right\|_{L^{p}\left(\mathbf{R}^{3}\right)} \geq\|u\|_{L^{p}\left(\mathbf{R}^{3} \times y\right)} \geq\|\hat{u}\|_{L^{p}\left(\mathbf{R}^{3}\right)} .
\end{gathered}
$$

If $u_{\varepsilon} \rightarrow \hat{u}$ in $L^{p}\left(\mathbf{R}^{3}\right)$ then $\hat{u}=u$, that is, $u$ does not depend on $y$.

Dealing with functions of $(x, y)$, we denote the gradient operator w.r.t. $x$ $(y$, resp. $)$ by $\nabla_{x}\left(\nabla_{y}\right.$, resp. $)$. We also set

$$
\begin{aligned}
& L_{\mathrm{rot}}^{2}\left(\mathbf{R}^{3}\right)^{3}:=\left\{\vec{v} \in L^{2}\left(\mathbf{R}^{3}\right)^{3}: \nabla \times \vec{v} \in L^{2}\left(\mathbf{R}^{3}\right)^{3}\right\}, \\
& L_{\mathrm{div}}^{2}\left(\mathbf{R}^{3}\right)^{3}:=\left\{\vec{v} \in L^{2}\left(\mathbf{R}^{3}\right)^{3}: \nabla \cdot \vec{v} \in L^{2}\left(\mathbf{R}^{3}\right)\right\}
\end{aligned}
$$

these are Hilbert spaces equipped with the respective graph norm. For any $v \in L_{\mathrm{loc}}^{1}\left(\mathbf{R}^{3} \times \mathcal{Y}\right)$ let us introduce its average and fluctuating components,

$$
\hat{v}(x):=\int_{y} v(x, y) d y, \quad \tilde{v}(x, y):=v(x, y)-\hat{v}(x) \text { for a.e. }(x, y) \in \mathbf{R}^{3} \times \mathcal{Y} .
$$

For any $p \in] 1,+\infty\left[\right.$ let us denote by $W_{*}^{1, p}(\mathscr{Y})$ the space of functions of $W^{1, p}(\mathcal{Y})$ having vanishing average; by the Poincaré inequality this is a Banach space equipped with the norm $\|v\|_{W_{*}^{1, p}(\mathscr{y})}:=\|\nabla v\|_{L^{p}(y)^{3}}$. We shall use the index $*$ throughout to denote subspaces that consist of functions of $y \in \mathcal{Y}$ having vanishing average, and also set $H_{*}^{1}(\mathcal{Y}):=W_{*}^{1,2}(\mathcal{Y})$.

The next three statements deal with the two-scale limit of derivatives; the first one is a basic result due to Nguetseng, the other two extend it.

Proposition 3.3 (Allaire, 1992; Nguetseng, 1989). Let $p \in] 1,+\infty[$, and a sequence $\left\{u_{\varepsilon}\right\}$ of $W^{1, p}\left(\mathbf{R}^{3}\right)$ be such that $u_{\varepsilon} \rightarrow u$ in $W^{1, p}\left(\mathbf{R}^{3}\right)$. Then there exists $u_{1} \in$ $L^{p}\left(\mathbf{R}^{3} ; W_{*}^{1, p}(\mathscr{Y})\right)$ such that, as $\varepsilon \rightarrow 0$ along a suitable subsequence,

$$
\nabla u_{\varepsilon} \underset{2}{\rightarrow} \nabla u+\nabla_{y} u_{1} \text { in } L^{p}\left(\mathbf{R}^{3} \times \mathscr{Y}\right)^{3} \text {. }
$$

Proposition 3.4 (Visintin, 2004, 2007a). Let $\left\{\vec{u}_{\varepsilon}\right\}$ be a bounded sequence of $L_{\mathrm{rot}}^{2}\left(\mathbf{R}^{3}\right)^{3}$ such that $\vec{u}_{\varepsilon} \underset{2}{\vec{u}}$ in $L^{2}\left(\mathbf{R}^{3} \times \mathscr{Y}\right)^{3}$. Then $\hat{\vec{u}} \in L_{\mathrm{rot}}^{2}\left(\mathbf{R}^{3}\right)^{3}$ and $\nabla_{y} \times \vec{u}=\overrightarrow{0}$ in $\mathscr{D}^{\prime}\left(\mathbf{R}^{3} \times \mathscr{Y}\right)^{3}$. Moreover there exists $\vec{u}_{1} \in L^{2}\left(\mathbf{R}^{3} ; H_{*}^{1}(\mathcal{Y})^{3}\right)$ such that $\nabla_{y} \cdot \vec{u}_{1}=0$ a.e. in $\mathbf{R}^{3} \times \mathcal{Y}$, and, as $\varepsilon \rightarrow 0$ along a suitable subsequence,

$$
\nabla \times \vec{u}_{\varepsilon} \underset{2}{\rightarrow} \nabla \times \hat{\vec{u}}+\nabla_{y} \times \vec{u}_{1} \text { in } L^{2}\left(\mathbf{R}^{3} \times Y\right)^{3} .
$$


Proposition 3.5 (Visintin, 2004, 2007a). Let $\left\{\vec{u}_{\varepsilon}\right\}$ be a bounded sequence of $L_{\mathrm{div}}^{2}\left(\mathbf{R}^{3}\right)^{3}$ such that $\vec{u}_{\varepsilon} \underset{2}{\vec{u}}$ in $L^{2}\left(\mathbf{R}^{3} \times \mathscr{Y}\right)^{3}$. Then $\hat{\vec{u}} \in L_{\mathrm{div}}^{2}\left(\mathbf{R}^{3}\right)^{3}$ and $\nabla_{y} \cdot \vec{u}=0$ in $\mathscr{D}^{\prime}\left(\mathbf{R}^{3} \times \mathscr{Y}\right)$. Moreover there exists $\vec{u}_{1} \in L^{2}\left(\mathbf{R}^{3} ; H_{*}^{1}(\mathcal{Y})^{3}\right)$ such that $\nabla_{y} \times \vec{u}_{1}=\overrightarrow{0}$ a.e. in $\mathbf{R}^{3} \times \mathcal{Y}$, and, as $\varepsilon \rightarrow 0$ along a suitable subsequence,

$$
\nabla \cdot \vec{u}_{\varepsilon} \underset{2}{\rightarrow} \nabla \cdot \hat{\vec{u}}+\nabla_{y} \cdot \vec{u}_{1} \text { in } L^{2}\left(\mathbf{R}^{3} \times \mathscr{Y}\right)
$$

These results are easily extended to functions defined on any Lipschitz subdomain of $\mathbf{R}^{3}$.

Two-Scale Div-Curl Lemma. First we state an extension of the (single-scale) div-curl lemma to time-dependent functions, that may be proved via a simple modification of the argument of Murat (1978).

Proposition 3.6 (Time-Dependent Div-Curl Lemma). Let $\left\{\vec{u}_{m}\right\}$ and $\left\{\vec{w}_{m}\right\}$ be two bounded sequences of $L^{2}\left(0, T ; L_{\mathrm{rot}}^{2}\left(\mathbf{R}^{3}\right)^{3}\right)$ and $L^{2}\left(0, T ; L_{\mathrm{div}}^{2}\left(\mathbf{R}^{3}\right)^{3}\right)$, resp.. If

$\exists r>0, \exists s \in \mathbf{R}$ such that either $\left\{\vec{u}_{m}\right\}$ or $\left\{\vec{w}_{m}\right\}$ is bounded in $H^{r}\left(0, T ; H^{s}\left(\mathbf{R}^{3}\right)^{3}\right)$,

$$
\vec{u}_{m} \rightarrow \vec{u}, \quad \vec{w}_{m} \rightarrow \vec{w} \quad \text { in } L^{2}\left(\mathbf{R}_{T}^{3}\right)^{3},
$$

then

$$
\iint_{\mathbf{R}_{T}^{3}} \vec{u}_{m}(x, t) \cdot \vec{w}_{m}(x, t) \theta(x, t) d x d t \rightarrow \iint_{\mathbf{R}_{T}^{3}} \vec{u}(x, t) \cdot \vec{w}(x, t) \theta(x, t) d x d t \quad \forall \theta \in \mathscr{D}\left(\mathbf{R}_{T}^{3}\right) .
$$

Here is a two-scale extension.

Proposition 3.7 (Two-Scale Time-Dependent Div-Curl Lemma) (Visintin, 2007b). Let $\left\{\vec{u}_{\varepsilon}\right\}$ be a bounded sequence of $L^{2}\left(0, T ; L_{\mathrm{rot}}^{2}\left(\mathbf{R}^{3}\right)^{3}\right)$, and $\left\{\vec{w}_{\varepsilon}\right\}$ be a bounded sequence of $L^{2}\left(0, T ; L_{\mathrm{div}}^{2}\left(\mathbf{R}^{3}\right)^{3}\right)$. If (3.9) holds and

$$
\vec{u}_{\varepsilon} \underset{2}{\vec{u}}, \quad \vec{w}_{\varepsilon} \underset{2}{\vec{w}} \text { in } L^{2}\left(\mathbf{R}_{T}^{3} \times \mathscr{Y}\right)^{3}
$$

then

$$
\begin{aligned}
& \iint_{\mathbf{R}_{T}^{3}} \vec{u}_{\varepsilon}(x, t) \cdot \vec{w}_{\varepsilon}(x, t) \theta(x, t) d x d t \rightarrow \iint_{\mathbf{R}_{T}^{3}} \hat{\vec{u}}(x, t) \cdot \hat{\vec{w}}(x, t) \theta(x, t) d x d t \\
& \quad=\iiint_{\mathbf{R}_{T}^{3} \times y} \vec{u}(x, y, t) \cdot \vec{w}(x, y, t) \theta(x, t) d x d y d t \quad \forall \theta \in \mathscr{D}\left(\mathbf{R}_{T}^{3}\right) .
\end{aligned}
$$

\section{Convexity, Monotonicity and Two-Scale Convergence}

In this section we gather some results about the two-scale behaviour of convex integral functionals and of maximal monotone graphs, in view of their use in the study of the electromagnetic problem of Sec. 2. For most of these results we provide two versions: first we deal with the customary single-scale convergence, and then consider the extension to two-scale convergence. Both versions will be needed in the sequel. In each case the single-scale formulation stems from classical properties of 
convexity and maximal monotonicity; the two-scale versions are less obvious, and are proved in Visintin (2007b). Here we deal with $\mathbf{R}^{3}$, however the statements that do not involve the curl operator hold unchanged in any $\mathbf{R}^{N}$.

We shall denote by $\mathscr{L}\left(\mathbf{R}^{3}\right)\left(\mathscr{B}\left(\mathbf{R}^{3}\right)\right.$, resp.) the $\sigma$-algebra of Lebesgue- (Borel-, resp.) measurable subsets of $\mathbf{R}^{3}$, define $\mathscr{L}(\Omega), \mathscr{B}(\Omega), \mathscr{L}(\mathscr{Y})$ and $\mathscr{B}(\mathscr{Y})$ similarly, and denote by $\mathscr{A}_{1} \otimes \mathscr{A}_{2}$ the $\sigma$-algebra generated by any pair $\mathscr{A}_{1}, \mathscr{A}_{2}$ of $\sigma$-algebras. We set

$$
\begin{aligned}
\mathscr{F}:=\{ & f: \mathbf{R}^{3} \times \Omega \times \mathscr{Y} \rightarrow \mathbf{R} \cup\{+\infty\} \text { measurable either w.r.t. } \mathscr{B}\left(\mathbf{R}^{3}\right) \\
& \otimes \mathscr{B}(\Omega) \otimes \mathscr{L}(\mathscr{Y}) \text { or w.r.t. } \mathscr{B}\left(\mathbf{R}^{3}\right) \otimes \mathscr{L}(\Omega) \otimes \mathscr{B}(\mathscr{Y}), \text { and } \\
& \left.\left\{\vec{v} \in \mathbf{R}^{3}: f(\vec{v}, x, y)<+\infty\right\} \text { has nonempty interior, for a.e. }(x, y)\right\} ;
\end{aligned}
$$

this class includes the Caratheodory functions. Each of these functions will be identified with its $Y$-periodic extension w.r.t. the third argument, consistently with our previous stipulation. In the next statement we gather some simple properties of integral functionals that will be used in the sequel.

Lemma 4.1 (Visintin, 2007b).

(i) Let $\varphi \in \mathscr{F}$. Then

$$
\begin{aligned}
& x \mapsto \varphi(\vec{v}(x), x, x / \varepsilon) \text { is measurable, for any measurable } \vec{v}: \Omega \rightarrow \mathbf{R}^{3}, \quad \forall \varepsilon>0, \\
& (x, y) \mapsto \varphi(\vec{v}(x, y), x, y) \text { is measurable, for any measurable } \vec{v}: \Omega \times \mathcal{Y} \rightarrow \mathbf{R}^{3}
\end{aligned}
$$

(ii) Moreover assume that

$$
\begin{aligned}
& \text { the function } v \mapsto \varphi(v, x, y) \text { is convex and lower semicontinuous } \\
& \text { for a.e. }(x, y), \\
& \exists \vec{w} \in L^{2}(\Omega)^{3}, \quad \exists h \in L^{1}(\Omega): \varphi(\vec{v}, x, y) \geq \vec{w}(x) \cdot \vec{v}+h(x) \\
& \forall \vec{v}, \quad \text { for a.e. }(x, y),
\end{aligned}
$$

and set

$$
\begin{aligned}
\varphi_{\varepsilon}(\vec{v}, x):=\varphi(\vec{v}, x, x / \varepsilon) \quad \forall(\vec{v}, x) \in \mathbf{R}^{3} \times \Omega, \quad \forall \varepsilon>0, \\
\Phi_{\varepsilon}: L^{2}(\Omega)^{3} \rightarrow \mathbf{R} \cup\{+\infty\}: \vec{v} \mapsto \int_{\Omega} \varphi_{\varepsilon}(\vec{v}(x), x) d x \quad \forall \varepsilon>0, \\
\Phi: L^{2}(\Omega \times y)^{3} \rightarrow \mathbf{R} \cup\{+\infty\}: \vec{v} \mapsto \iint_{\Omega \times y} \varphi(\vec{v}(x, y), x, y) d x d y .
\end{aligned}
$$

The functionals $\Phi_{\varepsilon}$ and $\Phi$ are then well-defined, convex and lower semicontinuous. (iii) Under the above hypotheses, for any sequence $\left\{\vec{v}_{\varepsilon}\right\}$ in $L^{2}(\Omega)^{3}$,

$$
\vec{v}_{\varepsilon} \underset{2}{\rightarrow} \vec{v} \text { in } L^{2}(\Omega \times Y)^{3} \Rightarrow \liminf _{\varepsilon \rightarrow 0} \Phi_{\varepsilon}\left(\vec{v}_{\varepsilon}\right) \geq \Phi(\vec{v}) .
$$

Parts (i) and (ii) stem from known properties of integral functionals. For the simple argument of part (iii) we refer to Visintin (2007b), where most of the results of this section are also proved. 
By standard notation we shall denote by $\varphi^{*}$ the Legendre-Fenchel convex conjugate function of $\varphi$ w.r.t. the first variable; see e.g., Ekeland and Temam (1974), Hiriart-Urruty and Lemarechal (1993), and Rockafellar (1969). The convex conjugate functionals $\Phi_{\varepsilon}^{*}$ and $\Phi^{*}$ are then the integral functionals of $\varphi_{\varepsilon}^{*}$ and $\varphi^{*}$, resp.; see e.g., Ekeland and Temam (1974) and Rockafellar (1968). We state the next results for (space- and) time-dependent functions with a parameter $\varepsilon>0$, in view of the use that we shall make afterwards.

Proposition 4.2. Let us fix any $\varepsilon>0$. Let $\varphi \in \mathscr{F}$ fulfil (4.3) and (4.4), define $\varphi_{\varepsilon}, \Phi_{\varepsilon}$ as in (4.5), and let $\left\{\vec{u}_{m}\right\}$ and $\left\{\vec{w}_{m}\right\}$ be sequences of $L^{2}\left(\Omega_{T}\right)^{3}$. If

$$
\begin{gathered}
\vec{w}_{m}(x, t) \in \partial \varphi_{\varepsilon}\left(\vec{u}_{m}(x, t), x\right) \text { for a.e. }(x, t) \in \Omega_{T}, \quad \forall m, \\
\vec{u}_{m} \rightarrow \vec{u}, \quad \vec{w}_{m} \rightarrow \vec{w} \quad \text { in } L^{2}\left(\Omega_{T}\right)^{3}, \\
\liminf _{m \rightarrow \infty} \iint_{\Omega_{T}} \vec{u}_{m} \cdot \vec{w}_{m} d x d t \leq \iint_{\Omega_{T}} \vec{u} \cdot \vec{w} d x d t
\end{gathered}
$$

then

$$
\begin{gathered}
\vec{w}(x, t) \in \partial \varphi_{\varepsilon}(\vec{u}(x, t), x) \text { for a.e. }(x, t) \in \Omega_{T} \\
\int_{0}^{T} \Phi_{\varepsilon}\left(\vec{u}_{m}\right) d t \rightarrow \int_{0}^{T} \Phi_{\varepsilon}(\vec{u}) d t, \quad \int_{0}^{T} \Phi_{\varepsilon}^{*}\left(\vec{w}_{m}\right) d t \rightarrow \int_{0}^{T} \Phi_{\varepsilon}^{*}(\vec{w}) d t \\
\iint_{\Omega_{T}} \vec{u}_{m} \cdot \vec{w}_{m} d x d t \rightarrow \iint_{\Omega_{T}} \vec{u} \cdot \vec{w} d x d t
\end{gathered}
$$

This statement may be proved via standard convexity techniques, cf. Theorem 2.1 of Visintin (2007b). Here is an extension to two-scale convergence.

Proposition 4.3 (Visintin, 2007b). Let $\varphi \in \mathscr{F}$ fulfil (4.3) and (4.4), and $\varphi_{\varepsilon}, \Phi_{\varepsilon}, \Phi$ be defined as in (4.5) for any $\varepsilon>0$. Let $\left\{\vec{u}_{\varepsilon}\right\}$ and $\left\{\vec{w}_{\varepsilon}\right\}$ be sequences of $L^{2}\left(\Omega_{T}\right)^{3}$. If

$$
\begin{gathered}
\vec{w}_{\varepsilon}(x, t) \in \partial \varphi_{\varepsilon}\left(\vec{u}_{\varepsilon}(x, t), x\right) \text { for a.e. }(x, t) \in \Omega_{T}, \forall \varepsilon, \\
\vec{u}_{\varepsilon} \overrightarrow{2} \vec{u}, \quad \vec{w}_{\varepsilon} \overrightarrow{2} \vec{w} \quad \text { in } L^{2}\left(\Omega_{T} \times Y\right)^{3}, \\
\liminf _{\varepsilon \rightarrow 0} \iint_{\Omega_{T}} \vec{u}_{\varepsilon}(x, t) \cdot \vec{w}_{\varepsilon}(x, t) d x d t \leq \iiint_{\Omega_{T} \times y} \vec{u}(x, y, t) \cdot \vec{w}(x, y, t) d x d y d t,
\end{gathered}
$$

then

$$
\begin{gathered}
\vec{w}(x, y, t) \in \partial \varphi(\vec{u}(x, y, t), x, y) \text { for a.e. }(x, y, t) \in \Omega_{T} \times \mathcal{Y}, \\
\int_{0}^{T} \Phi_{\varepsilon}\left(\vec{u}_{\varepsilon}\right) d t \rightarrow \int_{0}^{T} \Phi(\vec{u}) d t, \quad \int_{0}^{T} \Phi_{\varepsilon}^{*}\left(\vec{w}_{\varepsilon}\right) d t \rightarrow \int_{0}^{T} \Phi^{*}(\vec{w}) d t, \\
\iint_{\Omega_{T}} \vec{u}_{\varepsilon}(x, t) \cdot \vec{w}_{\varepsilon}(x, t) d x d t \rightarrow \iiint_{\Omega_{T} \times y} \vec{u}(x, y, t) \cdot \vec{w}(x, y, t) d x d y d t .
\end{gathered}
$$

Remark 4.4. It is easy to see that Proposition 4.3 also holds if a weight $\theta(x, t)$ is inserted into (4.15) and (4.18), for some nonnegative $\theta \in \mathscr{D}\left(\Omega_{T}\right)$, and $\Phi_{\varepsilon}$ and $\Phi$ are 
respectively replaced by the functionals

$$
v \mapsto \int_{\Omega} \varphi_{\varepsilon}(\vec{v}(x), x) \theta(x, t) d x, \quad v \mapsto \iint_{\Omega \times y} \varphi(\vec{v}(x, y), x, y) \theta(x, t) d x d y .
$$

In this case however (4.16) just holds for a.e. $(x, y, t) \in Q \times \mathcal{Y}, Q$ being the support of $\theta$. An analogous remark applies to Proposition 4.2.

\section{Compactness by Strict Convexity.}

Proposition 4.5 (Visintin, 1984, 1996, Chap. X). Let $\varphi \in \mathscr{F}$, and define $\varphi_{\varepsilon}$ as in $(4.5)_{1}$ for a fixed $\varepsilon>0$. Moreover assume that

$$
\begin{aligned}
\exists c>0, \exists h & \in L^{1}(\Omega): \forall \vec{v}, \text { for a.e. }(x, y) \in \Omega \times \mathcal{Y} \quad \varphi(\vec{v}, x, y) \geq c|\vec{v}|^{2}+h(x), \\
\vec{v} & \mapsto \varphi(\vec{v}, x, y) \text { is strictly convex, for a.e. }(x, y) \in \Omega \times \mathcal{Y} .
\end{aligned}
$$

For any sequence $\left\{\vec{u}_{m}\right\}$ of $L^{2}\left(\Omega_{T}\right)^{3}$, if

$$
\begin{gathered}
\vec{u}_{m} \rightarrow \vec{u} \quad \text { in } L_{\mathrm{loc}}^{2}\left(\Omega_{T}\right)^{3}, \\
\iint_{\Omega_{T}} \varphi_{\varepsilon}\left(\vec{u}_{m}(x, t), x\right) \theta(x, t) d x d t \rightarrow \iint_{\Omega_{T}} \varphi_{\varepsilon}(\vec{u}(x, t), x) \theta(x, t) d x d t \\
\forall \theta \in \mathscr{D}\left(\Omega_{T}\right),
\end{gathered}
$$

then

$$
\vec{u}_{m} \rightarrow \vec{u} \text { in } L_{\mathrm{loc}}^{2}\left(\Omega_{T}\right)^{3} .
$$

Here is an extension to two-scale convergence.

Proposition 4.6 (Visintin, 2007b). Let $\varphi \in \mathscr{F}$ fulfill (4.19), (4.20), and define $\varphi_{\varepsilon}$ as in $(4.5)_{1}$ for any $\varepsilon>0$. For any sequence $\left\{\vec{u}_{\varepsilon}\right\}$ of $L^{2}\left(\Omega_{T}\right)^{3}$, if

$$
\begin{gathered}
\vec{u}_{\varepsilon} \overrightarrow{2} \vec{u} \text { in } L_{\mathrm{loc}}^{2}\left(\Omega_{T} \times \mathcal{Y}\right)^{3}, \\
\iint_{\Omega_{T}} \varphi_{\varepsilon}\left(\vec{u}_{\varepsilon}(x, t), x\right) \theta(x, t) d x d t \rightarrow \iiint_{\Omega_{T} \times \mathscr{Y}} \varphi(\vec{u}(x, y, t), x, y) \theta(x, t) d x d y d t \\
\forall \theta \in \mathscr{D}\left(\Omega_{T}\right),
\end{gathered}
$$

then

$$
\vec{u}_{\varepsilon} \underset{2}{\rightarrow} \vec{u} \text { in } L_{\mathrm{loc}}^{2}\left(\Omega_{T} \times \mathscr{Y}\right)^{3}
$$

Monotonicity and Two-Scale Convergence. Next we partially extend the latter results to single-valued maximal monotone functions. First let us set

$$
\begin{aligned}
\mathscr{G}:=\left\{\vec{\alpha}:\left(\mathbf{R}^{3}\right)^{2} \times \Omega \times \mathscr{Y} \rightarrow \mathbf{R}^{3} \text { such that } \vec{\alpha}(\cdot, \cdot, x, y) \text { is continuous for a.e. }(x, y),\right. \\
\quad \text { and } \vec{\alpha}(\vec{v}, \vec{w}, \cdot, \cdot) \text { is measurable either w.r.t. } \mathscr{B}(\Omega) \otimes \mathscr{L}(\mathscr{Y}) \\
\quad \text { or w.r.t. } \mathscr{L}(\Omega) \times \mathscr{B}(\mathscr{Y}), \quad \forall(\vec{v}, \vec{w})\},
\end{aligned}
$$


let us fix a function $\vec{\alpha} \in \mathscr{G}$ such that

$$
\begin{aligned}
& \vec{v} \mapsto \vec{\alpha}(\vec{v}, \vec{w}, x, y) \text { is nondecreasing, for any } \vec{w} \text { and a.e. }(x, y), \\
& \exists L_{1}, L_{2}>0, \exists g_{1}, g_{2} \in L^{1}\left(\mathbf{R}^{3}\right): \forall \vec{v}, \forall \vec{w}, \quad \text { for a.e. }(x, y), \\
& L_{1}|\vec{v}|+g_{1}(x) \leq|\vec{\alpha}(\vec{v}, \vec{w}, x, y)| \leq L_{2}(|\vec{v}|+|\vec{w}|)+g_{2}(x),
\end{aligned}
$$

and define the Caratheodory functions

$$
\vec{\alpha}_{\varepsilon}(\vec{v}, \vec{w}, x):=\vec{\alpha}(\vec{v}, \vec{w}, x, x / \varepsilon) \quad \forall(\vec{v}, \vec{w}, x) \in\left(\mathbf{R}^{3}\right)^{2} \times \Omega, \forall \varepsilon>0 .
$$

The next statement extends a known result, cf. e.g., Brezis (1973, p. 27).

Proposition 4.7. Let $\varepsilon>0, \vec{\alpha} \in \mathcal{G}$ fulfil (4.28) and (4.29), and define $\vec{\alpha}_{\varepsilon}$ as in (4.30). Let $\left\{\vec{u}_{m}\right\}$ and $\left\{\vec{z}_{m}\right\}$ be sequences of $L^{2}\left(\Omega_{T}\right)^{3}$. If

$$
\begin{gathered}
\vec{w}_{m}(x, t)=\vec{\alpha}_{\varepsilon}\left(\vec{u}_{m}(x, t), \vec{z}_{m}(x, t), x\right) \quad \text { for a.e. }(x, t) \in \Omega_{T}, \forall m, \\
\vec{u}_{m} \rightarrow \vec{u}, \quad \vec{w}_{m} \rightarrow \vec{w}, \quad \vec{z}_{m} \rightarrow \vec{z} \text { in } L^{2}\left(\Omega_{T}\right)^{3}, \\
\liminf _{m \rightarrow \infty} \iint_{\Omega_{T}} \vec{u}_{m} \cdot \vec{w}_{m} d x d t \leq \iint_{\Omega_{T}} \vec{u} \cdot \vec{w} d x d t
\end{gathered}
$$

then

$$
\vec{w}(x, t)=\vec{\alpha}_{\varepsilon}(\vec{u}(x, t), \vec{z}(x, t), x) \text { for a.e. }(x, t) \in \Omega_{T} \text {. }
$$

Here is a two-scale extension, which may also be compared with Proposition 4.3.

Proposition 4.8 (Visintin, 2007b). Let $\vec{\alpha} \in \mathcal{G}$ fulfil (4.28), (4.29), and define $\vec{\alpha}_{\varepsilon}$ as in (4.30) for any $\varepsilon>0$. Let $\left\{\vec{u}_{\varepsilon}\right\}$ and $\left\{\vec{z}_{\varepsilon}\right\}$ be sequences of $L^{2}\left(\Omega_{T}\right)^{3}$. If

$$
\begin{array}{r}
\vec{w}_{\varepsilon}(x, t)=\vec{\alpha}_{\varepsilon}\left(\vec{u}_{\varepsilon}(x, t), \vec{z}_{\varepsilon}(x, t), x\right) \text { for a.e. }(x, t) \in \Omega_{T}, \forall \varepsilon, \\
\vec{u}_{\varepsilon} \overrightarrow{2} \vec{u}, \quad \vec{w}_{\varepsilon} \overrightarrow{2} \vec{w}, \quad \vec{z}_{\varepsilon} \underset{2}{\vec{z}} \text { in } L^{2}\left(\Omega_{T} \times Y\right)^{3}, \\
\liminf _{\varepsilon \rightarrow 0} \iint_{\Omega_{T}} \vec{u}_{\varepsilon}(x, t) \cdot \vec{w}_{\varepsilon}(x, t) d x d t \leq \iiint_{\Omega_{T} \times y} \vec{u}(x, y, t) \cdot \vec{w}(x, y, t) d x d y d t,
\end{array}
$$

then

$$
\vec{w}(x, y, t)=\vec{\alpha}(\vec{u}(x, y, t), \vec{z}(x, y, t), x, y) \text { for a.e. }(x, y, t) \in \Omega_{T} \times \mathcal{Y} .
$$

If $\vec{\alpha}$ is independent of its second argument, then the two latter results hold also if $\vec{\alpha}$ is multivalued.

Remark 4.9. It is easy to see that (4.38) also holds if (4.37) is replaced by

$$
\begin{aligned}
& \liminf _{\varepsilon \rightarrow 0} \iint_{\Omega_{T}} \vec{u}_{\varepsilon}(x, t) \cdot \vec{w}_{\varepsilon}(x, t) \theta(x, t) d x d t \\
& \quad \leq \iiint_{\Omega_{T} \times \mathscr{y}} \vec{u}(x, y, t) \cdot \vec{w}(x, y, t) \theta(x, t) d x d y d t \quad \forall \theta \in \mathscr{D}\left(\Omega_{T}\right), \theta \geq 0 .
\end{aligned}
$$

An analogous remark applies to Proposition 4.7. 


\section{A Parabolic-Hyperbolic Problem for an Inhomogeneous Material}

In this section we deal with electromagnetic processes in a periodic inhomogeneous material. We couple the Maxwell system with nonlinear constitutive relations of the form $\vec{B}=\vec{B}(\vec{H})$ and $\vec{J}=\vec{J}(\vec{E}, \vec{H})$, provide a weak formulation in Sobolev spaces, and prove the existence of a solution.

We assume that we are given a function $\varphi \in \mathscr{F}$ that fulfils (4.19), and a function $\vec{\alpha} \in \mathscr{G}$ that fulfils (4.28), (4.29) and such that

$$
\begin{gathered}
\exists c>0, \quad \exists h_{1} \in L^{1}(\Omega): \forall(\vec{v}, \vec{w}, x), \quad \text { for a.e. } y, \\
\vec{\alpha}(\vec{v}, \vec{w}, x, y) \cdot \vec{v} \geq c|\vec{\alpha}(\vec{v}, \vec{w}, x, y)|^{2}+c|\vec{v}|^{2}+h_{1}(x) .
\end{gathered}
$$

We extend these functions to $x \in \Omega^{\prime}\left(:=\mathbf{R}^{3} \backslash \Omega\right)$ by setting

$$
\begin{aligned}
& \varphi(\vec{v}, x, y):=\frac{|\vec{v}|^{2}}{2} \quad \forall(\vec{v}, \vec{w}, x, y) \in\left(\mathbf{R}^{3}\right)^{2} \times \Omega^{\prime} \times \mathcal{Y} . \\
& \vec{\alpha}(\vec{v}, \vec{w}, x, y):=0
\end{aligned}
$$

We select any $\varepsilon>0$ and keep it fixed throughout this section. We define $\varphi_{\varepsilon}$ and $\vec{\alpha}_{\varepsilon}$ as in (4.5) $)_{1}$ and (4.30), and assume that

$$
\begin{gathered}
\vec{E}_{\varepsilon}^{0} \in L^{2}\left(\Omega^{\prime}\right)^{3}, \quad \vec{B}_{\varepsilon}^{0} \in L^{2}\left(\mathbf{R}^{3}\right)^{3}, \quad \nabla \cdot \vec{B}_{\varepsilon}^{0}=0 \quad \text { in } \mathscr{D}^{\prime}\left(\mathbf{R}^{3}\right), \\
\left.\vec{g} \in L^{2}\left(\mathbf{R}_{T}^{3}\right)^{3}, \quad \nabla \cdot \vec{g}=0 \quad \text { in } \mathscr{D}^{\prime}\left(\mathbf{R}^{3}\right), \quad \text { a.e. in }\right] 0, T[.
\end{gathered}
$$

We are now able to introduce a weak formulation of the problem that we outlined in Sec. 2.

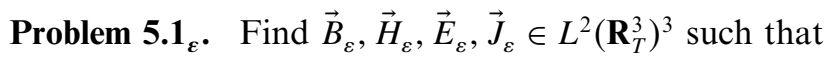

$$
\begin{gathered}
\iint_{\mathbf{R}_{T}^{3}}\left(\vec{H}_{\varepsilon} \cdot \nabla \times \vec{v}-\left(\vec{J}_{\varepsilon}+\vec{g}\right) \cdot \vec{v}+\left(1-\chi_{\Omega}\right)\left(\vec{E}_{\varepsilon}-\vec{E}_{\varepsilon}^{0}\right) \cdot \frac{\partial \vec{v}}{\partial t}\right) d x d t=0 \\
\forall \vec{v} \in H^{1}\left(\mathbf{R}_{T}^{3}\right)^{3}, \quad \vec{v}(\cdot, T)=\overrightarrow{0} \text { a.e. in } \mathbf{R}^{3} \\
\iint_{\mathbf{R}_{T}^{3}}\left(\vec{E}_{\varepsilon} \cdot \nabla \times \vec{v}+\left(\vec{B}_{\varepsilon}^{0}-\vec{B}_{\varepsilon}\right) \cdot \frac{\partial \vec{v}}{\partial t}\right) d x d t=0 \\
\forall \vec{v} \in H^{1}\left(\mathbf{R}_{T}^{3}\right)^{3}, \quad \vec{v}(\cdot, T)=\overrightarrow{0} \text { a.e. in } \mathbf{R}^{3}, \\
\vec{B}_{\varepsilon}(x, t) \in \partial \varphi_{\varepsilon}\left(\vec{H}_{\varepsilon}(x, t), x\right) \text { for a.e. }(x, t) \in \mathbf{R}_{T}^{3}, \\
\vec{J}_{\varepsilon}(x, t)=\vec{\alpha}_{\varepsilon}\left(\vec{E}_{\varepsilon}(x, t), \vec{H}_{\varepsilon}(x, t), x\right) \text { for a.e. }(x, t) \in \mathbf{R}_{T}^{3} .
\end{gathered}
$$

Interpretation. (5.5) and (5.6) respectively entail the Ampère-Maxwell and Faraday laws (2.1) and (2.2) (with normalized coefficients) in the form

$$
\begin{gathered}
\nabla \times \vec{H}_{\varepsilon}=\vec{J}_{\varepsilon}+\vec{g}+\left(1-\chi_{\Omega}\right) \frac{\partial \vec{E}_{\varepsilon}}{\partial t} \quad \text { in } \mathscr{D}^{\prime}\left(\mathbf{R}_{T}^{3}\right)^{3}, \\
\nabla \times \vec{E}_{\varepsilon}=-\frac{\partial \vec{B}_{\varepsilon}}{\partial t} \quad \text { in } \mathscr{D}^{\prime}\left(\mathbf{R}_{T}^{3}\right)^{3} .
\end{gathered}
$$


By comparing the terms of these equations we see that

$$
\vec{H}_{\varepsilon}, \vec{E}_{\varepsilon} \in H^{-1}\left(0, T ; L_{\mathrm{rot}}^{2}\left(\mathbf{R}^{3}\right)^{3}\right), \quad\left(1-\chi_{\Omega}\right) \vec{E}_{\varepsilon}, \quad \vec{B}_{\varepsilon} \in H^{1}\left(0, T ;\left(L_{\mathrm{rot}}^{2}\left(\mathbf{R}^{3}\right)^{3}\right)^{\prime}\right) ;
$$

therefore (5.9) and (5.10) hold in $\left(L_{\text {rot }}^{2}\left(\mathbf{R}^{3}\right)^{3}\right)^{\prime}$, a.e. in $] 0, T$ [. Integrating by parts in time in (5.5), (5.6), and using (5.9), (5.10), we then retrieve the initial conditions

$$
\vec{E}_{\varepsilon}(\cdot, 0)=\vec{E}_{\varepsilon}^{0} \quad \text { in } \mathscr{D}^{\prime}\left(\Omega^{\prime}\right)^{3}, \quad \vec{B}_{\varepsilon}(\cdot, 0)=\vec{B}_{\varepsilon}^{0} \quad \text { in } \mathscr{D}^{\prime}\left(\mathbf{R}^{3}\right)^{3} .
$$

In $\Omega_{T}$ the equation (5.9) is reduced to $\nabla \times \vec{H}_{\varepsilon}=\vec{J}_{\varepsilon}+\vec{g}$ in the sense of distributions; hence by (5.4)

$$
\nabla \cdot \vec{J}_{\varepsilon}=0 \text { in } \mathscr{D}^{\prime}\left(\Omega_{T}\right)^{3}
$$

Because of (5.2) the relation (5.7) is linear in $\Omega_{T}^{\prime}$. Thus Problem $5.1_{\varepsilon}$ is nonlinear parabolic in $\Omega_{T}$ and linear hyperbolic in $\Omega_{T}^{\prime}$.

Theorem 5.1. Let (5.1)-(5.4) hold, as well as (4.19), (4.20), (4.28) and (4.29). Moreover assume that

$$
\begin{aligned}
& \exists C>0, \quad \exists \tilde{h} \in L^{2}(\Omega): \forall \vec{v} \in \mathbf{R}^{3}, \quad \forall \vec{w} \in \partial \varphi(\vec{v}, x, y), \\
& |\vec{w}| \leq C|\vec{v}|+\tilde{h}(x) \text { for a.e. }(x, y),
\end{aligned}
$$

and let $\varphi_{\varepsilon}$ and $\vec{\alpha}_{\varepsilon}$ be defined as in (4.5), and (4.30). Then there exists a solution of Problem 5. $1_{\varepsilon}$ such that

$$
\begin{aligned}
& \vec{B}_{\varepsilon} \in L^{\infty}\left(0, T ; L^{2}\left(\mathbf{R}^{3}\right)^{3}\right), \\
& \vec{H}_{\varepsilon} \in L^{\infty}\left(0, T ; L^{2}\left(\mathbf{R}^{3}\right)^{3}\right),\left.\quad \vec{H}_{\varepsilon}\right|_{\Omega} \in L^{2}\left(0, T ; L_{\mathrm{rot}}^{2}(\Omega)^{3}\right), \\
& \vec{E}_{\varepsilon} \in L^{2}\left(\mathbf{R}_{T}^{3}\right)^{3}, \quad \vec{E}_{\varepsilon}\left(1-\chi_{\Omega}\right) \in L^{\infty}\left(0, T ; L^{2}\left(\Omega^{\prime}\right)^{3}\right) .
\end{aligned}
$$

Proof. Throughout this proof we drop the index $\varepsilon$ in order to render formulas more readable.

(i) Approximation. We fix any $m \in \mathbf{N}$, set $k:=T / m$ and

$$
\vec{B}_{m}^{0}:=\vec{B}^{0}, \quad \vec{E}_{m}^{0}:=\vec{E}^{0}, \quad \vec{g}_{m}^{n}:=\frac{1}{k} \int_{(n-1) k}^{n k} \vec{g}(\xi) d \xi \quad \text { for } n=1, \ldots, m
$$

We also fix any measurable selection $\vec{H}_{m}^{0}$ of $\partial \varphi_{\varepsilon}^{*}\left(\vec{B}_{m}^{0}, \cdot\right)$ (the specific choice of this selection turns out to be immaterial), and introduce an implicit time-discretization scheme of Problem 5.1.

Problem 5.1 $\mathbf{m}_{\mathrm{m}}$. Find $\vec{B}_{m}^{n} \in L^{2}\left(\mathbf{R}^{3}\right)^{3}, \quad \vec{E}_{m}^{n}, \vec{H}_{m}^{n} \in L_{\mathrm{rot}}^{2}\left(\mathbf{R}^{3}\right)^{3} \quad$ and $\quad \vec{J}_{m}^{n} \in L^{2}\left(\mathbf{R}^{3}\right)^{3} \quad(n=$ $1, \ldots, m)$, such that for $n=1, \ldots, m$

$$
\nabla \times \vec{H}_{m}^{n}=\vec{J}_{m}^{n}+\left(1-\chi_{\Omega}\right) \frac{\vec{E}_{m}^{n}-\vec{E}_{m}^{n-1}}{k}+\vec{g}_{m}^{n} \text { a.e. in } \mathbf{R}^{3}
$$




$$
\begin{gathered}
\nabla \times \vec{E}_{m}^{n}=\frac{\vec{B}_{m}^{n-1}-\vec{B}_{m}^{n}}{k} \text { a.e. in } \mathbf{R}^{3} \\
\vec{B}_{m}^{n}(x) \in \partial \varphi\left(\vec{H}_{m}^{n}(x), x\right) \text { for a.e. } x \in \mathbf{R}^{3} \\
\vec{J}_{m}^{n}(x)=\vec{\alpha}\left(\vec{E}_{m}^{n}(x), \vec{H}_{m}^{n-1}(x), x\right) \text { for a.e. } x \in \mathbf{R}^{3} .
\end{gathered}
$$

In view of proving existence of a solution of this problem, notice that (5.14)-(5.17) may be reduced to the following system:

$$
\begin{aligned}
& k \vec{\alpha}\left(\vec{E}_{m}^{n}, \vec{H}_{m}^{n-1}, x\right)+\left(1-\chi_{\Omega}\right) \vec{E}_{m}^{n}-k \nabla \times \vec{H}_{m}^{n}=\left(1-\chi_{\Omega}\right) \vec{E}_{m}^{n-1}-k \vec{g}_{m}^{n}=: \vec{f}_{m}^{n}, \\
& \quad \partial \varphi\left(\vec{H}_{m}^{n}, x\right)+k \nabla \times \vec{E}_{m}^{n} \ni \vec{B}_{m}^{n-1} \forall n .
\end{aligned}
$$

By eliminating the field $\vec{H}_{m}^{n}$, this also reads

$$
k \vec{\alpha}\left(\vec{E}_{m}^{n}, \vec{H}_{m}^{n-1}, x\right)+\left(1-\chi_{\Omega}\right) \vec{E}_{m}^{n}-k \nabla \times \partial \varphi^{*}\left(\vec{B}_{m}^{n-1}-k \nabla \times \vec{E}_{m}^{n}\right) \ni \vec{f}_{m}^{n}
$$

This inclusion is of the form $\mathscr{A}\left(\vec{E}_{m}^{n}\right)+\mathscr{B}\left(\vec{E}_{m}^{n}\right) \ni \vec{f}_{m}^{n}$, where the operator $\mathscr{A}(\mathscr{B}$, resp.): $L_{\text {rot }}^{2}\left(\mathbf{R}^{3}\right)^{3} \rightarrow\left(L_{\text {rot }}^{2}\left(\mathbf{R}^{3}\right)^{3}\right)^{\prime}$ is associated to the first (the sum of the second and third, resp.) addenda of (5.18). As $\vec{\alpha} \in \mathscr{G}$ and by (4.28), (4.29) and (5.1), it is easy to see that $\mathscr{A}$ is monotone, hemicontinuous and coercive; moreover $\mathscr{B}$ is maximal monotone for it is the subdifferential of a lower semicontinuous convex function. By known results (see e.g., Barbu, 1976; Brezis, 1973; Lions, 1969) $\mathscr{A}+\mathscr{B}$ is then maximal monotone. (5.18) thus has a solution $\vec{E}_{m}^{n}$, and this determines a solution of Problem $5.1_{\mathrm{m}}$.

(ii) A Priori Estimates. In view of rewriting time-discretized equations in continuous form, for any family $\left\{\phi_{m}^{n}\right\}_{n=0, \ldots, m}$ of functions $\Omega \rightarrow \mathbf{R}$ let us set

$$
\begin{aligned}
\phi_{m} & :=\text { piecewise-linear time-interpolate of } \phi_{m}^{0}, \ldots, \phi_{m}^{m}, \quad \text { a.e. in } \Omega, \\
\bar{\phi}_{m}(\cdot, t) & \left.:=\phi_{m}^{n} \quad \text { a.e. in } \Omega, \forall t \in\right](n-1) h, n h[, \quad \text { for } n=1, \ldots, m .
\end{aligned}
$$

We shall also use this notation for vector functions, and set $\overline{\vec{H}}_{m}(x, t):=\overrightarrow{0}$ for any $t<0$. The system (5.14)-(5.17) then reads

$$
\begin{gathered}
\nabla \times \overline{\vec{H}}_{m}=\overline{\vec{J}}_{m}+\left(1-\chi_{\Omega}\right) \frac{\partial \vec{E}_{m}}{\partial t}+\overline{\vec{g}}_{m} \text { a.e. in } \mathbf{R}_{T}^{3}, \\
\nabla \times \overline{\vec{E}}_{m}=-\frac{\partial \vec{B}_{m}}{\partial t} \text { a.e. in } \mathbf{R}_{T}^{3}, \\
\overline{\vec{B}}_{m}(x, t) \in \partial \varphi\left(\overline{\vec{H}}_{m}(x, t), x\right) \text { for a.e. }(x, t) \in \mathbf{R}_{T}^{3}, \\
\overrightarrow{\vec{J}}_{m}(x, t)=\vec{\alpha}\left(\overrightarrow{\vec{E}}_{m}(x, t), \overline{\vec{H}}_{m}(x, t-k), x\right) \text { for a.e. }(x, t) \in \mathbf{R}_{T}^{3} .
\end{gathered}
$$


Let us now multiply (5.20) by $\overline{\vec{E}}_{m},(5.21)$ by $-\overline{\vec{H}}_{m}$, sum these equalities, and integrate in time. By (5.16) we get

$$
\begin{gathered}
\int_{\mathbf{R}^{3}}\left[\varphi^{*}\left(\overline{\vec{B}}_{m}(\cdot, t)\right)-\varphi^{*}\left(\vec{B}^{0}\right)\right] d x+\iint_{\Omega_{t}} \overline{\overrightarrow{\vec{J}}}_{m} \cdot \overline{\vec{E}}_{m} d x+\frac{1}{2} \int_{\Omega^{\prime}}\left(\left|\vec{E}_{m}(\cdot, t)\right|^{2}-\left|\vec{E}^{0}\right|^{2}\right) d x \\
\left.=-\int_{0}^{t} d \tau \int_{\mathbf{R}^{3}} \overline{\vec{g}}_{m} \cdot \overline{\vec{E}}_{m} d x \leq \int_{0}^{t}\left\|\overline{\vec{g}}_{m}\right\|_{L^{2}\left(\mathbf{R}^{3}\right)^{3}}\left\|\overline{\vec{E}}_{m}\right\|_{L^{2}\left(\mathbf{R}^{3}\right)^{3}} d \tau \text { for a.e. } t \in\right] 0, T[
\end{gathered}
$$

By (4.19) and (5.1) a standard calculation then yields (omitting restrictions)

$$
\left\|\vec{E}_{m}\right\|_{L^{2}\left(\Omega_{T}\right)^{3} \cap L^{\infty}\left(0, T ; L^{2}\left(\Omega^{\prime}\right)^{3}\right.}, \quad\left\|\vec{B}_{m}\right\|_{L^{\infty}\left(0, T ; L^{2}\left(\mathbf{R}^{3}\right)^{3}\right)}, \quad\left\|\vec{J}_{m}\right\|_{L^{2}\left(\Omega_{T}\right)^{3}} \leq C_{1} .
$$

(By $C_{1}, C_{2}, \ldots$, we shall denote suitable constants independent of $m$.) Then by comparing the terms of (5.20) and (5.21) we also get

$$
\left\|\vec{H}_{m}\right\|_{L^{2}\left(0, T ; L_{\mathrm{rot}}^{2}(\Omega)^{3}\right)}, \quad\left\|\vec{B}_{m}\right\|_{H^{1}\left(0, T ;\left(L_{\mathrm{rot}}^{2}\left(\mathbf{R}^{3}\right)^{3}\right)^{\prime}\right)}, \quad\left\|\vec{E}_{m}\right\|_{H^{-1}\left(0, T ; L_{\mathrm{rot}}^{2}\left(\mathbf{R}^{3}\right)^{3}\right)} \leq C_{2} .
$$

(iii) Limit Procedure and Proof of (5.7). By the above estimates there exist $\vec{B}, \vec{H}, \vec{E}, \vec{J}$ such that, as $m \rightarrow \infty$ along a suitable sequence, (still omitting restrictions)

$$
\begin{gathered}
\vec{B}_{m} \stackrel{*}{\rightarrow} \vec{B} \text { in } L^{\infty}\left(0, T ; L^{2}\left(\mathbf{R}^{3}\right)^{3}\right) \cap H^{1}\left(0, T ;\left(L_{\mathrm{rot}}^{2}\left(\mathbf{R}^{3}\right)^{3}\right)^{\prime}\right), \\
\vec{H}_{m} \stackrel{*}{\rightarrow} \vec{H} \quad \text { in } L^{\infty}\left(0, T ; L^{2}\left(\mathbf{R}^{3}\right)^{3}\right) \cap L^{2}\left(0, T ; L_{\mathrm{rot}}^{2}(\Omega)^{3}\right), \\
\vec{E}_{m} \stackrel{*}{\rightarrow} \vec{E} \text { in } L^{2}\left(\Omega_{T}\right)^{3} \cap L^{\infty}\left(0, T ; L^{2}\left(\Omega^{\prime}\right)^{3}\right) \cap H^{-1}\left(0, T ; L_{\mathrm{rot}}^{2}\left(\mathbf{R}^{3}\right)^{3}\right), \\
\vec{J}_{m} \rightarrow \vec{J} \text { in } L^{2}\left(\Omega_{T}\right)^{3} .
\end{gathered}
$$

Passing to the limit in (5.20) and (5.21) we get (5.9) and (5.10). By (5.3) and (5.21), $\vec{B}_{m}$ is divergence-free. For any nonnegative $\theta \in \mathscr{D}\left(\Omega_{T}\right)$ by Proposition 3.6 we then have

$$
\iint_{\Omega_{T}} \overline{\vec{B}}_{m} \cdot \overline{\vec{H}}_{m} \theta d x d t \rightarrow \iint_{\Omega_{T}} \vec{B} \cdot \vec{H} \theta d x d t
$$

By Proposition 4.2 and Remark 4.4 we then get (5.7) and (still omitting the index $\varepsilon$ )

$$
\begin{aligned}
\iint_{\Omega_{T}} \varphi\left(\vec{H}_{m}(x, t), x\right) \theta(x, t) d x d t & \rightarrow \iint_{\Omega_{T}} \varphi(\vec{H}(x, t), x) \theta(x, t) d x d t, \\
\iint_{\Omega_{T}} \varphi^{*}\left(\vec{B}_{m}(x, t), x\right) \theta(x, t) d x d t & \rightarrow \iint_{\Omega_{T}} \varphi^{*}(\vec{B}(x, t), x) \theta(x, t) d x d t .
\end{aligned}
$$

By (5.1) Proposition 4.5 then yields

$$
\vec{H}_{m} \rightarrow \vec{H} \text { in } L_{\mathrm{loc}}^{2}\left(\Omega_{T}\right)^{3}
$$


(iii) Proof of (5.8). Let us now fix any $\zeta \in \mathscr{D}(\Omega)$, multiply (5.20) by $\overline{\vec{E}}_{m} \zeta$ and (5.21) by $-\overrightarrow{\vec{H}}_{m} \zeta$, sum these two formulas, and integrate in time. By (5.16) we get

$$
\begin{aligned}
& \int_{\Omega} {\left[\varphi^{*}\left(\vec{B}_{m}(\cdot, t)\right)-\varphi^{*}\left(\vec{B}^{0}\right)\right] \zeta d x+\iint_{\Omega_{t}}\left(\overline{\overrightarrow{\vec{g}}}_{m}+\overline{\vec{J}}_{m}\right) \cdot \overline{\vec{E}}_{m} \zeta d x d \tau } \\
&\left.=\iint_{\Omega_{t}} \overline{\vec{H}}_{m} \times \overline{\vec{E}}_{m} \cdot \nabla \zeta d x d \tau \text { for a.e. } t \in\right] 0, T[
\end{aligned}
$$

We would like to operate similarly with the limit equations (5.9) and (5.10), but miss the necessary regularity for $\vec{E} \zeta$ to do so. In view of removing this drawback, first we fix any $\lambda>0$, set

$$
\rho_{\lambda}(x):=(\lambda \pi)^{-3 / 2} \exp \left(-|x|^{2} / \lambda\right) \quad \forall x \in \mathbf{R}^{3}, \quad \vec{v}_{\lambda}:=\vec{v} * \rho_{\lambda} \quad \forall \vec{v} \in L^{2}\left(\mathbf{R}^{3}\right)^{3},
$$

and convolute the equations (5.9) and (5.10) with $\rho_{\lambda}$. We then multiply these equations respectively by $\vec{E}_{\lambda} \zeta$ and $-\vec{H}_{\lambda} \zeta$, sum them and integrate in time. This yields (still omitting the index $\varepsilon$ )

$$
\begin{gathered}
\iint_{\Omega_{t}} \frac{\partial \vec{B}_{\lambda}}{\partial t} \cdot \vec{H}_{\lambda} \zeta d x d \tau+\iint_{\Omega_{t}}\left(\vec{g}_{\lambda}+\vec{J}_{\lambda}\right) \cdot \vec{E}_{\lambda} \zeta d x d \tau \\
\left.\quad=\iint_{\Omega_{t}} \vec{H}_{\lambda} \times \vec{E}_{\lambda} \cdot \nabla \zeta d x d \tau \text { for a.e. } t \in\right] 0, T[
\end{gathered}
$$

Let us denote the support of $\zeta$ by $\tilde{\Omega}$, and notice that (omitting restrictions)

$$
\vec{H}_{\lambda} \rightarrow \vec{H} \quad \text { in } L^{2}\left(0, T ; L_{\text {rot }}^{2}(\tilde{\Omega})^{3}\right), \quad \vec{B}_{\lambda} \rightarrow \vec{B} \quad \text { in } H^{1}\left(0, T ;\left(L_{\text {rot }}^{2}(\tilde{\Omega})^{3}\right)^{\prime}\right)
$$

Denoting by $\langle\cdot, \cdot\rangle$ the duality pairing between $L_{\text {rot }}^{2}(\tilde{\Omega})^{3}$ and its dual, as $\lambda \rightarrow 0$ by (5.7) we then have

$$
\iint_{\Omega_{t}} \frac{\partial \vec{B}_{\lambda}}{\partial t} \cdot \vec{H}_{\lambda} \zeta d x d \tau \rightarrow \int_{0}^{t}\left\langle\frac{\partial \vec{B}}{\partial t}, \vec{H} \zeta\right\rangle d \tau=\int_{\Omega}\left[\varphi^{*}(\vec{B}(\cdot, t))-\varphi^{*}\left(\vec{B}^{0}\right)\right] \zeta d x,
$$

and by letting $\lambda$ vanish in (5.36) we get

$$
\begin{aligned}
& \int_{\Omega}\left[\varphi^{*}(\vec{B}(\cdot, t))-\varphi^{*}\left(\vec{B}^{0}\right)\right] \zeta d x+\iint_{\Omega_{t}}(\vec{g}+\vec{J}) \cdot \vec{E} \zeta d x d \tau=\iint_{\Omega_{t}} \vec{H} \times \vec{E} \cdot \nabla \zeta d x d \tau \\
& \quad \text { for a.e. } t \in] 0, T[.
\end{aligned}
$$

(Notice that the regularity of the solution allows us to write this equality.) Let us now pass to the inferior limit as $m \rightarrow+\infty$ in (5.34). By (5.27)-(5.29), (5.32) and (5.33) we have

$$
\begin{aligned}
& \int_{\Omega}\left[\varphi^{*}(\vec{B}(\cdot, t))-\varphi^{*}\left(\vec{B}^{0}\right)\right] \zeta d x+\iint_{\Omega_{t}} \vec{g} \cdot \vec{E} \zeta d x d \tau+\liminf _{m \rightarrow+\infty} \iint_{\Omega_{t}} \overline{\vec{J}}_{m} \cdot \overline{\vec{E}}_{m} \zeta d x d t \\
& \left.\quad \leq \iint_{\Omega_{t}} \vec{H} \times \vec{E} \cdot \nabla \zeta d x d \tau \text { for a.e. } t \in\right] 0, T[
\end{aligned}
$$


by comparing (5.37) and (5.38) we then get

$$
\left.\liminf _{m \rightarrow+\infty} \iint_{\Omega_{t}} \overline{\vec{J}}_{m} \cdot \overline{\vec{E}}_{m} \zeta d x d t \leq \iint_{\Omega_{t}} \vec{J} \cdot \vec{E} \zeta d x d t \text { for a.e. } t \in\right] 0, T[
$$

By (5.33) and by the maximal monotonicity of $\vec{\alpha}_{\varepsilon}$ w.r.t. its first argument, the constitutive relation (5.8) then follows, because of the extension of Proposition 4.7 that we pointed out in Remark 4.9.

Remark 5.2. By the above argument it is clear that the estimates (5.25) and (5.26) are also uniform w.r.t. $\varepsilon$. The same then applies to the limit functions; that is, displaying the index $\varepsilon$ but still omitting restrictions,

$$
\begin{aligned}
& \left\|\vec{B}_{\varepsilon}\right\|_{L^{\infty}\left(0, T ; L^{2}\left(\mathbf{R}^{3}\right)^{3}\right) \cap H^{1}\left(0, T ;\left(L_{\mathrm{rot}}^{2}\left(\mathbf{R}^{3}\right)^{3}\right)^{\prime}\right)}, \quad\left\|\vec{H}_{\varepsilon}\right\|_{L^{\infty}\left(0, T ; L^{2}\left(\mathbf{R}^{3}\right)^{3}\right) \cap L^{2}\left(0, T ; L_{\mathrm{rot}}^{2}(\Omega)^{3}\right)}, \\
& \left\|\vec{E}_{\varepsilon}\right\|_{L^{2}\left(\Omega_{T}\right)^{3} \cap L^{\infty}\left(0, T ; L^{2}\left(\Omega^{\prime}\right)^{3}\right)}, \quad\left\|\vec{J}_{\varepsilon}\right\|_{L^{2}\left(\Omega_{T}\right)^{3}} \leq \text { Constant independent of } \varepsilon .
\end{aligned}
$$

\section{Two-Scale Homogenization}

In this section we introduce a two-scale homogenized problem, that we then retrieve as the two-scale limit of the parabolic-hyperbolic Problem $5.1_{\varepsilon}$ as $\varepsilon \rightarrow 0$.

We assume that (5.1)-(5.4) are fulfilled, as well as (4.19), (4.20), (4.28), (4.29) and (5.12). We also require that

$$
\vec{B}_{\varepsilon}^{0} \underset{2}{\rightarrow} \vec{B}^{0} \quad \text { in } L^{2}\left(\mathbf{R}^{3} \times \mathscr{Y}\right)^{3}, \quad \vec{E}_{\varepsilon}^{0} \underset{2}{\rightarrow} \vec{E}^{0} \text { in } L^{2}\left(\Omega^{\prime} \times \mathscr{Y}\right)^{3} .
$$

Using the notation (3.5), let us also define two Hilbert subspaces of $L^{2}(\mathscr{Y})^{3}$ :

$$
\begin{array}{ll}
W:=\left\{\vec{w} \in L^{2}(\mathscr{Y})^{3}: \nabla \cdot \vec{w}=0 \text { in } \mathscr{D}^{\prime}(\mathscr{Y})\right\}, \quad W_{*}:=\{\vec{v} \in W: \hat{\vec{v}}=\overrightarrow{0}\}, \\
Z:=\left\{\vec{v} \in L^{2}(\mathscr{Y})^{3}: \nabla \times \vec{v}=\overrightarrow{0} \text { in } \mathscr{D}^{\prime}(\mathscr{Y})^{3}\right\}, \quad Z_{*}:=\{\vec{w} \in Z: \hat{\vec{w}}=\overrightarrow{0}\} .
\end{array}
$$

Next we introduce the weak formulation of a two-scale problem.

Problem 6.1. Find $\vec{H}, \vec{E} \in L^{2}\left(\mathbf{R}_{T}^{3} ; Z\right), \vec{B}, \vec{H}_{1}, \vec{E}_{1}, \vec{J} \in L^{2}\left(\mathbf{R}_{T}^{3} ; W\right)$ such that

$$
\begin{gathered}
\widehat{\vec{H}}_{1}=\widehat{\vec{E}}_{1}=\overrightarrow{0} \text { a.e. in } \mathbf{R}_{T}^{3}, \\
\iiint_{\mathbf{R}_{T}^{3} \times y}\left(\widehat{\vec{H}} \cdot \nabla_{x} \times \vec{v}+\vec{H}_{1} \cdot \nabla_{y} \times \vec{v}-(\vec{J}+\vec{g}) \cdot \vec{v}\right. \\
\left.+\left(1-\chi_{\Omega}\right)\left(\vec{E}-\vec{E}^{0}\right) \cdot \frac{\partial \vec{v}}{\partial t}\right) d x d y d t=0 \\
\forall \vec{v} \in H^{1}\left(\mathbf{R}_{T}^{3} \times \mathcal{Y}\right)^{3},\left.\vec{v}\right|_{t=T}=\overrightarrow{0} \quad \text { a.e. in } \mathbf{R}^{3} \times \mathcal{Y}, \\
\iiint_{\mathbf{R}_{T}^{3} \times y}\left(\widehat{\vec{E}} \cdot \nabla_{x} \times \vec{v}+\vec{E}_{1} \cdot \nabla_{y} \times \vec{v}+\left(\vec{B}^{0}-\vec{B}\right) \cdot \frac{\partial \vec{v}}{\partial t}\right) d x d y d t=0 \\
\forall \vec{v} \in H^{1}\left(\mathbf{R}_{T}^{3} \times \mathcal{Y}\right)^{3},\left.\vec{v}\right|_{t=T}=\overrightarrow{0} \quad \text { a.e. in } \mathbf{R}^{3} \times \mathcal{Y},
\end{gathered}
$$




$$
\begin{gathered}
\vec{B} \in \partial \varphi(\vec{H}, x, y) \quad \text { a.e. in } \mathbf{R}_{T}^{3} \times \mathcal{Y}, \\
\vec{J}=\vec{\alpha}(\vec{E}, \vec{H}, x, y) \quad \text { a.e. in } \mathbf{R}_{T}^{3} \times \mathcal{Y} .
\end{gathered}
$$

Interpretation. The equations (6.4) and (6.5) yield

$$
\begin{gathered}
\nabla \times \widehat{\vec{H}}+\nabla_{y} \times \vec{H}_{1}=\vec{J}+\vec{g}+\left(1-\chi_{\Omega}\right) \frac{\partial \vec{E}}{\partial t} \quad \text { in } \mathscr{D}^{\prime}\left(\mathbf{R}_{T}^{3} \times \mathscr{Y}\right)^{3}, \\
\nabla \times \widehat{\vec{E}}+\nabla_{y} \times \vec{E}_{1}=-\frac{\partial \vec{B}}{\partial t} \text { in } \mathscr{D}^{\prime}\left(\mathbf{R}_{T}^{3} \times \mathscr{Y}\right)^{3} .
\end{gathered}
$$

By (5.3) $\vec{B}^{0} \in W$. As $\vec{B}, \vec{J} \in W$ a.e. in $\mathbf{R}_{T}^{3}$, taking the divergence w.r.t. $x$ of the two latter equations we then get

$$
\begin{gathered}
\nabla \cdot \widehat{\vec{B}}=0 \quad \text { in } \mathscr{D}^{\prime}\left(\mathbf{R}_{T}^{3}\right)^{3}, \quad \nabla_{y} \cdot \vec{B}=0 \quad \text { in } \mathscr{D}^{\prime}\left(\mathbf{R}_{T}^{3} \times \mathscr{Y}\right)^{3}, \\
\left.\nabla \cdot \widehat{\vec{J}}=0 \quad \text { in } \mathscr{D}^{\prime}\left(\mathbf{R}^{3}\right)^{3}, \quad \nabla_{y} \cdot \vec{J}=0 \text { in } \mathscr{D}^{\prime}\left(\mathbf{R}^{3} \times \mathscr{Y}\right)^{3} \quad \text { a.e. in }\right] 0, T[.
\end{gathered}
$$

By comparing the terms of the equations (6.8) and (6.9) we see that

$$
\left(1-\chi_{\Omega}\right) \frac{\partial \vec{E}}{\partial t}, \frac{\partial \vec{B}}{\partial t} \in L^{2}\left(0, T ; H^{-1}\left(\mathbf{R}^{3} \times \mathcal{Y}\right)^{3}\right) .
$$

By integrating by parts in time in (6.4) and (6.5), we then get the initial conditions

$$
\left.\vec{E}\right|_{t=0}=\vec{E}^{0} \quad \text { in } \mathscr{D}^{\prime}\left(\Omega^{\prime} \times \mathscr{Y}\right)^{3},\left.\quad \vec{B}\right|_{t=0}=\vec{B}^{0} \quad \text { in } \mathscr{D}^{\prime}\left(\mathbf{R}^{3} \times \mathscr{Y}\right)^{3}
$$

Coarse-Scale Equations. By selecting test functions $\vec{v}$ independent of $y$ in (6.8) and (6.9) (formally, by integrating these equations over $\mathscr{Y}$ ), we retrieve the AmpèreMaxwell and Faraday laws (2.1) and (2.2) for the coarse-scale fields $\widehat{\vec{B}}, \widehat{\vec{H}}, \widehat{\vec{E}}, \widehat{\vec{J}}$ :

$$
\begin{gathered}
\nabla \times \widehat{\vec{H}}=\widehat{\vec{J}}+\left(1-\chi_{\Omega}\right) \frac{\partial \widehat{\vec{E}}}{\partial t}+\vec{g} \quad \text { in } \mathscr{D}^{\prime}\left(\mathbf{R}_{T}^{3}\right)^{3}, \\
\nabla \times \widehat{\vec{E}}=-\frac{\partial \overrightarrow{\vec{B}}}{\partial t} \text { in } \mathscr{D}^{\prime}\left(\mathbf{R}_{T}^{3}\right)^{3} .
\end{gathered}
$$

Moreover (6.10) yields the initial conditions

$$
\left.\widehat{\vec{E}}\right|_{t=0}=\widehat{\overrightarrow{E^{0}}} \quad \text { in } \mathscr{D}^{\prime}\left(\Omega^{\prime}\right)^{3},\left.\quad \widehat{\vec{B}}\right|_{t=0}=\widehat{\vec{B}^{0}} \text { in } \mathscr{D}^{\prime}\left(\mathbf{R}^{3}\right)^{3} .
$$

Fine-Scale Equations. By comparing the equations (6.8) and (6.9) with (6.11) and (6.12), we get (using the notation (3.5))

$$
\begin{gathered}
\nabla_{y} \times \vec{H}_{1}=\widetilde{\vec{J}}+\left(1-\chi_{\Omega}\right) \frac{\partial \widetilde{\vec{E}}}{\partial t} \quad \text { in } \mathscr{D}^{\prime}\left(\mathbf{R}_{T}^{3} \times \mathscr{Y}\right)^{3}, \\
\nabla_{y} \times \vec{E}_{1}=-\frac{\partial \tilde{\vec{B}}}{\partial t} \text { in } \mathscr{D}^{\prime}\left(\mathbf{R}_{T}^{3} \times \mathscr{Y}\right)^{3} .
\end{gathered}
$$


Similarly (6.10) and (6.13) yield the initial conditions

$$
\left.\widetilde{\vec{E}}\right|_{t=0}=\widetilde{\vec{E}}{ }^{0} \text { in } \mathscr{D}^{\prime}\left(\Omega^{\prime} \times \mathscr{Y}\right)^{3},\left.\quad \widetilde{\vec{B}}\right|_{t=0}=\widetilde{\vec{B}^{0}} \quad \text { in } \mathscr{D}^{\prime}\left(\mathbf{R}^{3} \times \mathscr{Y}\right)^{3} .
$$

By projecting the two-scale equations (6.8) and (6.9) on the space of $y$-independent functions, we have thus derived the equations (6.11) and (6.12) for the coarsescale fields; the equations (6.14) and (6.15) for the fine-scale fields then followed. The coarse- and fine-scale fields are coupled via the nonlinear constitutive equations (6.6) and (6.7).

Alike Problem 5.1 , Problem 6.1 is nonlinear parabolic in $\Omega$ and linear hyperbolic in $\Omega^{\prime}$. Next we retrieve this two-scale model from Problem $5.1_{\varepsilon}$ by passing to the two-scale limit as $\varepsilon$ vanishes.

Theorem 6.1. Let (4.19), (4.20), (4.28), (4.29), (5.1)-(5.4), (5.12), (6.1) be fufilled; let $\varphi_{\varepsilon}$ and $\vec{\alpha}_{\varepsilon}$ be then defined as in (4.5), and (4.30). For any $\varepsilon>0$, let $\left(\vec{B}_{\varepsilon}, \vec{H}_{\varepsilon}, \vec{E}_{\varepsilon}, \vec{J}_{\varepsilon}\right)$ be a solution of Problem 5. $1_{\varepsilon}$ (which exists after Theorem 5.1). If this family of solutions fulfils the uniform estimates (5.39) (by Remark 5.2 such a family exists), then there exist $\vec{B}, \vec{H}, \vec{E}, \vec{J}$ such that, as $\varepsilon \rightarrow 0$ along a suitable sequence,

$$
\begin{aligned}
& \vec{B}_{\varepsilon} \underset{2}{\stackrel{*}{\longrightarrow}} \vec{B}, \quad \vec{H}_{\varepsilon} \underset{2}{\stackrel{*}{\longrightarrow}} \vec{H} \text { in } L^{\infty}\left(0, T ; L^{2}\left(\mathbf{R}^{3} \times \mathcal{Y}\right)^{3}\right), \\
& \vec{E}_{\varepsilon} \underset{2}{\stackrel{*}{\longrightarrow}} \vec{E} \text { in } L^{2}\left(\Omega_{T} \times \mathscr{Y}\right)^{3} \cap L^{\infty}\left(0, T ; L^{2}\left(\Omega^{\prime} \times \mathscr{Y}\right)^{3}\right) \text {, } \\
& \vec{J}_{\varepsilon} \underset{2}{\rightarrow} \vec{J} \text { in } L^{2}\left(\Omega_{T} \times Y\right)^{3} .
\end{aligned}
$$

Moreover there exist $\vec{H}_{1}, \vec{E}_{1}$ such that $\left(\vec{B}, \vec{H}, \vec{E}, \vec{J}, \vec{H}_{1}, \vec{E}_{1}\right)$ is a solution of Problem 6.1 .

Proof. By Proposition 3.1 and by the estimates (5.39), there exist $\vec{B}, \vec{H}, \vec{E}, \vec{J}$ such that, as $\varepsilon \rightarrow 0$ along a suitable subsequence, (6.17)-(6.19) hold. Let us recall the definition (6.2) of the spaces $W, Z$. As $\nabla \times \vec{E}_{\varepsilon}$ is uniformly bounded in $L^{2}\left(\mathbf{R}_{T}^{3}\right)^{3}$, we infer that $\nabla_{y} \times \vec{E}=\overrightarrow{0}$ in $\mathscr{D}^{\prime}\left(\mathbf{R}_{T}^{3} \times \mathscr{Y}\right)^{3}$; therefore $\vec{E} \in L^{2}\left(\mathbf{R}_{T}^{3} ; Z\right)$. Similarly, $\nabla \cdot \vec{B}_{\varepsilon}=0$ a.e. in $\mathbf{R}_{T}^{3}$ and $\nabla \cdot \vec{J}_{\varepsilon}$ is uniformly bounded in $L^{2}\left(\mathbf{R}_{T}^{3}\right)$, hence $\nabla_{y} \cdot \vec{B}=\nabla_{y} \cdot \vec{J}=0$ in $\mathscr{D}^{\prime}\left(\mathbf{R}_{T}^{3} \times \mathscr{Y}\right)$; thus $\vec{B}, \vec{J} \in L^{2}\left(\mathbf{R}_{T}^{3} ; W\right)$.

After Proposition 3.4, by passing to the limit in (5.5) and (5.6), we get (6.4) and (6.5). For any nonnegative $\theta \in \mathscr{D}\left(\Omega_{T}\right)$, by Proposition 3.7 we have

$$
\iint_{\Omega_{T}} \vec{B}_{\varepsilon}(x, t) \cdot \vec{H}_{\varepsilon}(x, t) \theta(x, t) d x d t \rightarrow \iiint_{\Omega_{T} \times y} \vec{B}(x, y, t) \cdot \vec{H}(x, y, t) \theta(x, t) d x d y d t
$$

by Proposition 4.3 and Remark 4.4 this yields (6.6) and

$$
\begin{aligned}
\iint_{\Omega_{T}} \varphi_{\varepsilon}\left(\vec{H}_{\varepsilon}(x, t), x\right) \theta(x, t) d x d t & \rightarrow \iiint_{\Omega_{T} \times y} \varphi(\vec{H}(x, y, t), x, y) \theta(x, t) d x d y d t, \\
\iint_{\Omega_{T}} \varphi_{\varepsilon}^{*}\left(\vec{B}_{\varepsilon}(x, t), x\right) \theta(x, t) d x d t & \rightarrow \iiint_{\Omega_{T} \times y} \varphi^{*}(\vec{B}(x, y, t), x, y) \theta(x, t) d x d y d t .
\end{aligned}
$$


Obviously the latter convergence then also holds for any $\theta \in \mathscr{D}\left(\Omega_{T}\right)$ without sign restriction. By Proposition 4.6 we then get

$$
\vec{H}_{\varepsilon} \underset{2}{\rightarrow} \vec{H} \text { in } L_{\mathrm{loc}}^{2}\left(\Omega_{T} \times \mathcal{Y}\right)^{3}
$$

whence by (6.18)

$$
\vec{H}_{\varepsilon} \times \vec{E}_{\varepsilon} \underset{2}{\rightarrow} \vec{H} \times \vec{E} \text { in } L_{\mathrm{loc}}^{1}\left(\Omega_{T} \times \mathscr{Y}\right)^{3} .
$$

In order to prove (6.7) we shall proceed along the lines of the final step of the proof of Theorem 5.1. Let us define $\rho_{\lambda}=\rho_{\lambda}(x)$ and $\vec{v}_{\lambda}$ as in (5.35) for any $\vec{v} \in L^{2}\left(\mathbf{R}^{3}\right)$, and convolute the equations (6.8) and (6.9) with $\rho_{\lambda}$; we thus get two equations that we label by $(6.7)_{\lambda}$ and $(6.9)_{\lambda}$. Let us fix any nonnegative $\zeta \in \mathscr{D}(\Omega)$ (with support $\widetilde{\Omega}$, say) and any nonnegative $\eta \in \mathscr{D}(0, T)$ (with support $\left[t_{1}, t_{2}\right]$, say). As $\vec{E}, \vec{H} \in W$ a.e. in $\mathbf{R}_{T}^{3}$ then $\vec{E}_{\lambda}, \vec{H}_{\lambda} \in W$, so that we may multiply $(6.8)_{\lambda}$ and $(6.9)_{\lambda}$ by $\vec{E}_{\lambda} \zeta(x) \eta(t)$ and $-\vec{H}_{\lambda} \zeta(x) \eta(t)$, respectively. By summing these two formulas and integrating in time, we get

$$
\begin{aligned}
& \iiint_{\Omega_{T} \times y}\left(\frac{\partial \vec{B}_{\lambda}}{\partial t} \cdot \vec{H}_{\lambda} \zeta(x) \eta(t)+\left(\vec{g}_{\lambda}+\vec{J}_{\lambda}\right) \cdot \vec{E}_{\lambda} \zeta(x) \eta(t)\right) d x d y d t \\
& \quad=\iiint_{\Omega_{T} \times y} \vec{H}_{\lambda} \times \vec{E}_{\lambda} \cdot \nabla \zeta(x) \eta(t) d x d y d t .
\end{aligned}
$$

As $\lambda \rightarrow 0$,

$$
\begin{aligned}
& \vec{H}_{\lambda} \rightarrow \vec{H} \quad \text { in } L^{2}\left(\mathscr{Y}_{T} ; L_{\text {rot }}^{2}(\tilde{\Omega})^{3}\right), \\
& \frac{\partial \vec{B}_{\lambda}}{\partial t} \rightarrow \frac{\partial \vec{B}}{\partial t} \text { in } L^{2}\left(\mathscr{Y}_{T} ;\left(L_{\text {rot }}^{2}(\tilde{\Omega})^{3}\right)^{\prime}\right) .
\end{aligned}
$$

Denoting by $\langle\cdot, \cdot\rangle$ the duality pairing between $\mathscr{D}\left(\Omega_{T}\right)^{3}$ and its dual, by (6.6) we then have

$$
\begin{aligned}
& \iint_{\Omega_{T}} \frac{\partial \vec{B}_{\lambda}}{\partial t} \cdot \vec{H}_{\lambda} \zeta(x) \eta(t) d x d t \rightarrow\left\langle\frac{\partial}{\partial t} \varphi^{*}(\vec{B}(x, y, t), x, y), \zeta(x) \eta(t)\right\rangle \\
& \quad=-\iiint_{\Omega_{T} \times y} \varphi^{*}(\vec{B}(x, y, t), x, y) \zeta(x) \eta^{\prime}(t) d x d y d t ;
\end{aligned}
$$

by passing to the limit in (6.24) we then get

$$
\begin{aligned}
& \iiint_{\Omega_{T} \times y}\left[-\varphi^{*}(\vec{B}(x, y, t), x, y) \zeta(x) \eta^{\prime}(t)+(\vec{g}+\vec{J}) \cdot \vec{E} \zeta(x) \eta(t)\right] d x d y d t \\
& \quad=\iiint_{\Omega_{T} \times y} \vec{H} \times \vec{E} \cdot \nabla \zeta(x) \eta(t) d x d y d t .
\end{aligned}
$$

By operating similarly on the solution of Problem $5.1_{\varepsilon}$ we obtain

$$
\begin{aligned}
& \iint_{\Omega_{T}}\left[-\varphi_{\varepsilon}^{*}\left(\vec{B}_{\varepsilon}(x, t), x, y\right) \zeta(x) \eta^{\prime}(t)+\left(\vec{g}+\vec{J}_{\varepsilon}\right) \cdot \vec{E}_{\varepsilon} \zeta(x)\right] d x d t \\
& \quad=\iint_{\Omega_{T}} \vec{H}_{\varepsilon} \times \vec{E}_{\varepsilon} \cdot \nabla \zeta(x) \eta(t) d x d t \quad \forall \varepsilon
\end{aligned}
$$


We now pass to the inferior limit as $\varepsilon \rightarrow 0$ in the latter formula, apply (6.21) with $\theta(x, t)=\zeta(x) \eta^{\prime}(t)$ and also recall (6.23). Comparing the outcome with (6.25) we obtain

$$
\begin{aligned}
& \liminf _{\varepsilon \rightarrow 0} \iint_{\Omega_{T}} \vec{J}_{\varepsilon}(x, t) \cdot \vec{E}_{\varepsilon}(x, t) \zeta(x) \eta(t) d x d t \\
& \quad \leq \iiint_{\Omega_{T} \times y} \vec{J}(x, y, t) \cdot \vec{E}(x, y, t) \zeta(x) \eta(t) d x d y d t
\end{aligned}
$$

for any nonnegative $\zeta \in \mathscr{D}(\Omega)$ and any nonnegative $\eta \in \mathscr{D}(0, T)$. By Proposition 4.8 and Remark 4.9 the constitutive relation (6.7) then follows.

\section{Single-Scale Homogenization}

In this section we derive a coarse-scale homogenized problem by upscaling the two-scale Problem 6.1.

In view of the application of Propositions 7.1 and 7.2, here we assume that $\vec{\alpha}$ is cyclically monotone w.r.t. the first argument; that is,

$$
\vec{\alpha}(\vec{J}, \vec{E}, x, y)=\partial \psi(\vec{J}, \vec{E}, x, y) \quad \forall \vec{J}, \vec{E} \in \mathbf{R}^{3}, \quad \text { for a.e. }(x, y)
$$

where $\psi: \mathbf{R}^{3} \times \mathbf{R}^{3} \times \Omega \times \mathbf{R}^{3} \rightarrow \mathbf{R}$ is a prescribed function such that

$$
\begin{aligned}
& \psi \text { is measurable either w.r.t. } \mathscr{B}\left(\mathbf{R}^{3} \times \mathbf{R}^{3}\right) \otimes \mathscr{B}(\Omega) \otimes \mathscr{L}(\mathscr{Y}), \\
& \text { or w.r.t. } \mathscr{B}\left(\mathbf{R}^{3} \times \mathbf{R}^{3}\right) \otimes \mathscr{L}(\Omega) \otimes \mathscr{B}(\mathscr{Y}), \\
& \vec{v} \mapsto \psi(\vec{v}, \vec{w}, x, y) \text { is convex and lower semicontinuous, } \\
& \quad \text { for any } \vec{w} \text { and a.e. }(x, y), \\
& \left\{\vec{v} \in \mathbf{R}^{3}: \psi(\vec{v}, \vec{w}, x, y)<+\infty\right\} \text { has nonempty interior, } \\
& \quad \text { for any } \vec{w} \text { and a.e. }(x, y), \\
& \vec{w} \mapsto \psi(\vec{v}, \vec{w}, x, y) \text { is continuous, for any } \vec{v} \text { and a.e. }(x, y), \\
& y \mapsto \psi(\vec{v}, \vec{w}, x, y) \text { is Y-periodic, for any } \vec{v}, \vec{w} \text { and a.e. }(x, y) \text {. }
\end{aligned}
$$

Notice that the Hall law is not consistent with (7.1), because of the asymmetry of the tensor $\mathscr{A}$, cf. (2.12). Afterwards we shall also outline a modified setting in order to deal with that law.

We still assume that $\varphi \in \mathscr{F}$ (cf. (4.1)) and fulfils (4.19) and (4.20). Recalling the definition (6.2) of $Z_{*}$ we set

$$
\begin{gathered}
\varphi_{0}(\vec{H}, x):=\inf _{\vec{\eta} \in Z_{*}} \int_{y} \varphi(\vec{H}+\vec{\eta}(y), x, y) d y \quad \forall \vec{H} \in \mathbf{R}^{3}, \quad \text { for a.e. } x \in \Omega . \\
\psi_{0}(\vec{E}, \vec{H}, x):=\inf _{\vec{\eta} \in Z_{*}} \int_{y} \psi(\vec{E}+\vec{\eta}(y), \vec{H}, x, y) d y \quad \forall \vec{E}, \vec{H} \in \mathbf{R}^{3}, \quad \text { for a.e. } x \in \Omega .
\end{gathered}
$$


These minimization problems are obviously equivalent to the respective cell problems:

$$
\begin{gathered}
\left\{\begin{array}{l}
v_{1} \in H^{1}(\mathcal{Y})^{3} \\
\nabla_{y} \cdot \partial \varphi\left(\vec{H}+\nabla_{y} v_{1}(y), x, y\right) \ni 0 \text { in } H^{-1}(\mathscr{Y})^{3}
\end{array} \quad \text { for a.e. } x \in \Omega .\right. \\
\left\{\begin{array}{l}
v_{2} \in H^{1}(\mathscr{Y})^{3} \\
\nabla_{y} \cdot \partial \psi\left(\vec{E}+\nabla_{y} v_{2}(y), \vec{H}, x, y\right) \ni 0 \text { in } H^{-1}(\mathscr{Y})^{3}
\end{array} \quad \forall \vec{H} \in \mathbf{R}^{3} \text {, for a.e. } x \in \Omega .\right.
\end{gathered}
$$

Next we introduce a single-scale homogenized problem.

Problem 7.1. Find $\overline{\vec{B}}, \overline{\vec{H}}, \overrightarrow{\vec{E}}, \overline{\vec{J}} \in L^{2}\left(\mathbf{R}_{T}^{3}\right)^{3}$ such that

$$
\begin{gathered}
\iint_{\mathbf{R}_{T}^{3}}\left(\overline{\vec{H}} \cdot \nabla \times \vec{v}-(\overline{\vec{J}}+\vec{g}) \cdot \vec{v}+\left(1-\chi_{\Omega}\right)\left(\overline{\vec{E}}-\overline{\vec{E}}^{0}\right) \cdot \frac{\partial \vec{v}}{\partial t}\right) d x d t=0 \\
\forall \vec{v} \in H^{1}\left(\mathbf{R}_{T}^{3}\right)^{3}, \vec{v}(\cdot, T)=\overrightarrow{0} \quad \text { a.e. in } \mathbf{R}^{3}, \\
\iint_{\mathbf{R}_{T}^{3}}\left(\overline{\vec{E}} \cdot \nabla \times \vec{v}+\left(\widehat{\vec{B}}^{0}-\overline{\vec{B}}\right) \cdot \frac{\partial \vec{v}}{\partial t}\right) d x d t=0 \\
\forall \vec{v} \in H^{1}\left(\mathbf{R}_{T}^{3}\right)^{3}, \vec{v}(\cdot, T)=\overrightarrow{0} \text { a.e. in } \mathbf{R}^{3}, \\
\overline{\vec{B}} \in \partial \varphi_{0}(\overline{\vec{H}}, x) \text { a.e. in } \Omega_{T}, \quad \overline{\vec{B}}=\overline{\vec{H}} \text { a.e. in } \Omega_{T}^{\prime}, \\
\overline{\vec{J}} \in \partial \psi_{0}(\overline{\vec{E}}, \overline{\vec{H}}, x) \quad \text { a.e. in } \Omega_{T}, \overline{\vec{J}}=\overrightarrow{0} \text { a.e. in } \Omega_{T}^{\prime} .
\end{gathered}
$$

This problem differs from Problem $5.1_{\varepsilon}$ just in the constitutive functions (apart from the fact that here $\vec{\alpha}$ is assumed to be a subdifferential). By Theorem 5.1 it thus has a solution; the same conclusion will be attained via homogenization.

The next two Propositions will allow us to relate Problems 6.1 and 7.1 each other. We shall apply them to constitutive law in which the coarse-scale variable $x$ also occurs as a parameter. We state these results omitting the variable $x$, for the extension to the more general setting exhibits no additional difficulty, and refer the reader to Visintin (in preparation) for the more detailed formulation and argument. The next statement may be compared with classical results about the homogenization of integral functionals of Carbone and Sbordone (1979) and Marcellini (1978).

Proposition 7.1 (Visintin, in preparation). Let $\varphi: \mathbf{R}^{3} \times \mathcal{Y} \rightarrow \mathbf{R} \cup\{+\infty\}$ be a normal integrand, assume that $\varphi \in \mathscr{F}$ fulfils (4.3) and (4.4) (here without dependence on $x$ ), and set

$$
\varphi_{0}(\vec{\xi}):=\inf \left\{\int_{y} \varphi(\vec{\xi}+\vec{z}(y), y) d y: \vec{z} \in Z_{*}\right\} \forall \vec{\xi} \in \mathbf{R}^{3}
$$

For any $\vec{u} \in Z$ and $\vec{w} \in W$, if

$$
\vec{w}(y) \in \partial \varphi(\vec{u}(y), y) \text { for a.e. } y \in \mathscr{Y},
$$


then

$$
\hat{\vec{w}} \in \partial \varphi_{0}(\hat{\vec{u}})
$$

Proof. First notice that $Z_{*}=\nabla H_{*}^{1}(\mathscr{Y})$ and this is the orthogonal subspace of $V_{*}$ in $L^{2}(\mathscr{Y})^{3}$. Therefore

$$
\begin{aligned}
& \int_{y} \vec{v}(y) \cdot \vec{w}(y) d y-\hat{\vec{v}} \cdot \hat{\vec{w}}=\int_{y}[\hat{\vec{v}} \cdot \tilde{\vec{w}}(y)+\tilde{\vec{v}}(y) \cdot \hat{\vec{w}}+\tilde{\vec{v}}(y) \cdot \tilde{\vec{w}}(y)] d y \\
& \quad=\hat{\vec{v}} \cdot \int_{y} \tilde{\vec{w}}(y) d y+\left(\int_{y} \tilde{\vec{v}}(y) d y\right) \cdot \hat{\vec{w}}+\int_{y} \tilde{\vec{v}}(y) \cdot \tilde{\vec{w}}(y) d y=0 \quad \forall(\vec{v}, \vec{w}) \in Z \times W
\end{aligned}
$$

By (7.13)

$$
\int_{\mathscr{y}} \vec{w}(y) \cdot[\vec{u}(y)-\vec{v}(y)] d y \geq \int_{\mathscr{y}} \varphi(\vec{u}(y), y) d y-\int_{\mathscr{y}} \varphi(\vec{v}(y), y) d y \quad \forall \vec{v} \in L^{2}(\mathscr{Y})^{3} ;
$$

hence, applying (7.15) and noticing that $\int_{y} \varphi(\vec{u}(y), y) d y \geq \varphi_{0}(\hat{\vec{u}})$,

$$
\hat{\vec{w}} \cdot(\hat{\vec{u}}-\hat{\vec{v}})=\int_{y} \vec{w}(y) \cdot[\vec{u}(y)-\vec{v}(y)] d y \geq \varphi_{0}(\hat{\vec{u}})-\int_{y} \varphi(\vec{v}(y), y) d y \quad \forall \vec{v} \in Z
$$

By the arbitrariness of $\tilde{\vec{v}}$ we then get $\hat{\vec{w}} \cdot(\hat{\vec{u}}-\hat{\vec{v}}) \geq \varphi_{0}(\hat{\vec{u}})-\varphi_{0}(\hat{\vec{v}})$ for any $\hat{\vec{v}} \in \mathbf{R}^{3}$, that is (7.13).

Under further restrictions the latter result may be inverted. No information are thus lost by replacing (7.12) with the average inclusion (7.13).

Proposition 7.2 (Visintin, in preparation). Let $\varphi$ and $\varphi_{0}$ be as in Proposition 7.1. Assume that $\varphi$ is convex and lower semicontinuous, and that

$$
\varphi(\vec{v}, \cdot) \rightarrow+\infty, \quad \varphi^{*}(\vec{v}, \cdot) \rightarrow+\infty \text { as }|\vec{v}| \rightarrow+\infty, \quad \text { uniformly in } \mathcal{Y}
$$

If $\vec{r}, \vec{s} \in \mathbf{R}^{3}$ are such that $\vec{s} \in \partial \varphi_{0}(\vec{r})$, then there exist $\vec{u}, \vec{w} \in L^{2}(\mathscr{Y})^{3}$ such that

$$
\hat{\vec{u}}=\vec{r}, \quad \hat{\vec{w}}=\vec{s}, \quad \vec{w}(y) \in \partial \varphi(\vec{u}(y), y) \text { for a.e. } y \in \mathcal{Y} \text {. }
$$

If $\varphi(\cdot, y)$ and $\varphi^{*}(\cdot, y)$ are strictly convex for a.e. $y \in \mathcal{Y}$, then the pair $(\vec{u}, \vec{w})$ is unique.

Proof. Let us first set

$$
\psi_{0}(\vec{w}):=\inf \left\{\int_{\mathscr{y}} \varphi^{*}(\vec{w}+\vec{z}(y), y) d y: \vec{z} \in Z\right\} \quad \forall \vec{w} \in \mathbf{R}^{3}
$$


By the convexity and the lower semicontinuity of $\varphi$ and $\varphi^{*}$, the infima $\varphi_{0}(\hat{\vec{u}})$ and $\psi_{0}(\hat{\vec{w}})$ (cf. (7.11) and (7.18)) are both attained, that is,

$$
\begin{array}{ll}
\exists \vec{u} \in \mathbf{R}^{N}+W: \quad \hat{\vec{u}}=\vec{r}, \quad \varphi_{0}(\hat{\vec{u}})=\int_{y} \varphi(\vec{u}(y), y) d y, \\
\exists \vec{w} \in \mathbf{R}^{N}+Z: \quad \hat{\vec{w}}=\vec{s}, \quad \psi_{0}(\hat{\vec{w}})=\int_{y} \varphi^{*}(\vec{w}(y), y) d y .
\end{array}
$$

The inclusion $\vec{s} \in \partial \varphi_{0}(\vec{r})$ is tantamount to $\vec{r} \cdot \vec{s} \geq \varphi_{0}(\vec{r})+\varphi_{0}^{*}(\vec{s})$, namely by (7.19)

$$
\hat{\vec{u}} \cdot \hat{\vec{w}} \geq \varphi_{0}(\hat{\vec{u}})+\varphi_{0}^{*}(\hat{\vec{w}})
$$

The definitions of $\varphi_{0}$ and $\psi_{0}$ respectively yield $\varphi_{0} \leq \varphi$ and $\psi_{0} \leq \varphi^{*}$ in $\mathbf{R}^{3} \times \mathscr{Y}$; the former inequality entails that $\varphi^{*} \leq \varphi_{0}^{*}$. Thus $\psi_{0} \leq \varphi^{*} \leq \varphi_{0}^{*}$, namely $\psi_{0} \leq \varphi_{0}^{*}$ in $\mathbf{R}^{3}$. The inequality (7.20) then yields $\hat{\vec{u}} \cdot \hat{\vec{w}} \geq \varphi_{0}(\hat{\vec{u}})+\psi_{0}(\hat{\vec{w}})$, that by (7.14) and (7.19) reads

$$
\int_{\mathscr{y}} \vec{u}(y) \cdot \vec{w}(y) d y \geq \int_{\mathscr{y}} \varphi(\vec{u}(y), y) d y+\int_{\mathscr{y}} \varphi^{*}(\vec{w}(y), y) d y .
$$

By the definition of convex conjugate function on the other hand $\vec{u}(y) \cdot \vec{w}(y) \leq$ $\varphi(\vec{u}(y), y)+\varphi^{*}(\vec{w}(y), y)$ for a.e. $y \in \mathcal{Y}$. By the two latter inequalities we infer that

$$
\vec{u}(y) \cdot \vec{w}(y)=\varphi(\vec{u}(y), y)+\varphi^{*}(\vec{w}(y), y) \quad \text { for a.e. } y \in \mathscr{Y}
$$

by a well-known characterization of subdifferentials (cf. e.g., Ekeland and Temam, 1974; Sec. I.5) this is tantamount to $\vec{w}(y) \in \partial \varphi(\vec{u}(y), y)$ for a.e. $y \in \mathscr{Y}$. The final statement about uniqueness is obvious.

We can now establish a precise relation between Problems 6.1 and 7.1.

Theorem 7.3. Assume the hypotheses of Theorem 6.1 , and let $\left(\vec{B}, \vec{H}, \vec{E}, \vec{H}_{1}, \vec{E}, \vec{J}\right)$ be a solution of Problem 6.1 (that exists by Theorem 6.1). Assume that (7.1), (7.2) are also satisfied and set (7.3), (7.4). The average fields $\widehat{\vec{B}}, \widehat{\vec{H}}, \widehat{\vec{E}}, \widehat{\vec{J}}$ then solve Problem 7.1.

Conversely, for any solution $(\overrightarrow{\vec{B}}, \overrightarrow{\vec{H}}, \overline{\vec{E}}, \overline{\vec{J}})$ of Problem 7.1 there exists a solution $\left(\vec{B}, \vec{H}, \vec{E}, \vec{H}_{1}, \vec{E}_{1}, \vec{J}\right)$ of Problem 6.1 such that $\widehat{\vec{B}}=\overline{\vec{B}}, \overline{\vec{H}}=\overline{\vec{H}}, \widehat{\vec{E}}=\overline{\vec{E}}, \widehat{\vec{J}}=\overline{\vec{J}}$ a.e. in $\mathbf{R}_{T}^{3}$.

Proof. We already saw that (6.4) and (6.5) entail the coarse-scale equations (6.11)(6.13), that is (7.7) and (7.8). By Proposition 7.1 the relations (6.6) and (6.7) yield (7.9) and (7.10). The second statement directly follows from Proposition 7.2.

Theorems 6.1 and 7.3 yield the next result.

Corollary 7.4. Assume the hypotheses of Theorem 6.1, let (7.1), (7.2) be also satisfied, and set (7.3), (7.4). For any $\varepsilon>0$, let $\left(\vec{B}_{\varepsilon}, \vec{H}_{\varepsilon}, \vec{E}_{\varepsilon}, \vec{J}_{\varepsilon}\right)$ be a solution of Problem $5.1_{\varepsilon}$ (which exists after Theorem 5.1). Then there exist $\overrightarrow{\vec{B}}, \overrightarrow{\vec{H}}, \overrightarrow{\vec{E}}, \overrightarrow{\vec{J}}$ such that, as $\varepsilon \rightarrow 0$ along a suitable sequence,

$$
\vec{B}_{\varepsilon} \stackrel{*}{\rightarrow} \overrightarrow{\vec{B}}, \quad \vec{H}_{\varepsilon} \stackrel{*}{\rightarrow} \overline{\vec{H}} \quad \text { in } L^{\infty}\left(0, T ; L^{2}\left(\mathbf{R}^{3}\right)^{3}\right)
$$




$$
\begin{gathered}
\vec{E}_{\varepsilon} \stackrel{*}{\rightarrow} \overline{\vec{E}} \text { in } L^{2}\left(\Omega_{T}\right)^{3} \cap L^{\infty}\left(0, T ; L^{2}\left(\Omega^{\prime}\right)^{3}\right), \\
\vec{J}_{\varepsilon} \underset{2}{\overrightarrow{\vec{J}}} \text { in } L^{2}\left(\Omega_{T}\right)^{3} .
\end{gathered}
$$

This entails that $(\overline{\vec{B}}, \overline{\vec{H}}, \overline{\vec{E}}, \overline{\vec{J}})$ is a solution of Problem 7.1.

Single-Scale Homogenization of a Modified Maxwell-Hall Problem. In Sec. 2 we represented the Hall effect in full generality by (2.12), and in simplified form by (2.13). The latter relation is included in the more general (7.10), that we then considered in our analysis.

Setting $\mathscr{C}(\vec{H}):=\widetilde{\mathscr{A}}(\vec{H})^{-1},(2.6)$ (with $\left.\vec{g}=0\right)$ and (2.13) yield

$$
\vec{E}=\mathscr{C}(\vec{H}) \cdot \vec{J}-\vec{E}_{a}=\mathscr{C}(\vec{H}) \cdot \nabla \times \vec{H}-\vec{E}_{a} \text { a.e. in } \Omega_{T},
$$

for an applied electromotive force $\vec{E}_{a}$. In the two-scale framework we then get

$$
\begin{gathered}
\vec{E}(x, y, t)=\mathscr{C}(\vec{H}(x, y, t), x, y, t) \cdot\left(\nabla \times \hat{\vec{H}}(x, t)+\nabla_{y} \times \vec{H}_{1}(x, y, t)\right)-\vec{E}_{a}(x, t) \\
\quad \text { for a.e. }(x, y, t) \in \Omega_{T} \times \mathscr{Y} .
\end{gathered}
$$

Here we shall make the homogenized constitutive relation explicit for a simplified version of (7.25). We replace $\vec{H}(x, y, t)$ by the average field $\widehat{\vec{H}}(x, t)$ in the tensor $\mathfrak{b}$ :

$$
\begin{aligned}
\vec{E}(x, y, t)= & \mathscr{C}(\widehat{\vec{H}}(x, t), x, y, t) \cdot\left(\nabla \times \widehat{\vec{H}}(x, t)+\nabla_{y} \times \vec{H}_{1}(x, y, t)\right)-\vec{E}_{a}(x, t) \\
& \text { for a.e. }(x, y, t) \in \Omega_{T} \times \mathcal{Y} .
\end{aligned}
$$

This may be retrieved by replacing the relation (5.8) in $\Omega_{T}$ by

$$
\vec{E}_{\varepsilon}=\mathscr{C}\left(\int_{\mathscr{B}(x, \varepsilon)} \vec{H}_{\varepsilon}(\xi, t) d \xi, x, y, t\right) \cdot \vec{J}_{\varepsilon}-\vec{E}_{a} \quad \text { for a.e. }(x, t) \in \Omega_{T}
$$

$(\mathscr{B}(x, \varepsilon)$ denoting the ball of center $x$ and radius $\varepsilon)$ in Problem $5.1_{\varepsilon}$. This author ignores whether it is physically acceptable to replace the Hall law by this averaged formulation; anyway (7.26) obviously includes the case in which the resistivity tensor $\mathscr{b}$ is independent of the magnetic field. The developments of Secs. 5, 6 take over to this setting without any difficulty.

Next we make explicit the form of the homogenized conductivity tensor $\mathscr{C}_{0}$ along the lines of Allaire (1992); see also the appendix of Hornung (1997) and e.g., the works Bensoussan et al. (1978), Cioranescu and Donato (1999), Murat and Tartar (1997), and Sanchez-Palencia (1980). Let us assume that $\mathscr{C}$ is a positivedefinite tensor, and that $\mathscr{C}_{i j}$ a Caratheodory function for any $i, j$. The condition $\vec{E} \in Z$ a.e. in $\Omega_{T}$ (cf. (6.2)) reads

$$
\begin{aligned}
\nabla_{y} \times \vec{E}(x, y, t)= & \nabla_{y} \times\left\{\mathscr{C}(\widehat{\vec{H}}(x, t), x, y, t) \cdot\left[\nabla \times \widehat{\vec{H}}(x, t)+\nabla_{y} \times H_{1}(x, y, t)\right]\right\}=\overrightarrow{0} \\
& \text { in } H^{-1}(\mathscr{Y})^{3}, \quad \text { for a.e. }(x, t) \in \Omega_{T} .
\end{aligned}
$$


We claim that (7.26) and (7.27) entail

$$
\left.\widehat{\vec{E}}(x, t)=\mathscr{C}_{0} \widehat{\widehat{\vec{H}}}(x, t), x, t\right) \cdot \nabla \times \widehat{\vec{H}}(x, t)-\vec{E}_{a}(x, t) \text { for a.e. }(x, t) \in \Omega_{T},
$$

where the homogenized tensor $\mathscr{C}_{0}$ is determined as follows. Let us denote by $\vec{e}_{i}$ the unit vector of the axis $y_{i}$ for $i=1,2,3$, set $v_{i}:=\vec{v} \cdot \vec{e}_{i}$ for any $\vec{v}$, and search for a family of functions $\vec{w}_{1}(x, y, t), \ldots, \vec{w}_{3}(x, y, t)$ such that

$$
\vec{H}_{1}(x, y, t)=\sum_{i=1,2,3} \vec{w}_{i}(x, y, t)(\nabla \times \widehat{\vec{H}})_{i}(x, t) \text { for a.e. }(x, y, t) \in \Omega_{T} \times \mathcal{Y} .
$$

The equation (7.27) then reads

$$
\begin{gathered}
\nabla_{y} \times\left\{\mathscr{C}(\widehat{\vec{H}}(x, t), x, y, t) \cdot \sum_{i=1,2,3}(\nabla \times \widehat{\vec{H}})_{i}(x, t)\left[\vec{e}_{i}+\nabla_{y} \times \vec{w}_{i}(x, y, t)\right]\right\}=\overrightarrow{0} \\
\quad \text { in } H^{-1}(y)^{3}, \quad \text { for a.e. }(x, t) \in \Omega_{T},
\end{gathered}
$$

and this holds whenever $\vec{w}_{1}, \ldots, \vec{w}_{3}$ solve the next three cell problems:

$$
\begin{array}{r}
\nabla_{y} \times\left\{\mathscr{C}(\widehat{\vec{H}}(x, t), x, y, t) \cdot\left[\vec{e}_{i}+\nabla_{y} \times \vec{w}_{i}(x, y, t)\right]\right\}=\overrightarrow{0} \\
\text { in } H^{-1}(\mathscr{y})^{3}, \quad \text { for a.e. }(x, t) \in \Omega_{T}, \quad \text { for } i=1,2,3
\end{array}
$$

This family of elliptic problems has one and only one solution $\vec{w}_{1}, \ldots, \vec{w}_{3} \in$ $L^{2}\left(\Omega_{T} ; H_{*}^{1}(\mathscr{y})^{3}\right)$ such that $\nabla_{y} \cdot \vec{w}_{i}=0$ in $H^{-1}(\mathscr{Y})$, for a.e. $(x, t) \in \Omega_{T}$ and for $i=1,2,3$. By setting

$$
\begin{aligned}
\mathscr{C}_{0 i j}(v, x, t) & =\int_{y} \vec{e}_{i} \cdot \mathscr{C}(\widehat{\vec{H}}(x, t), x, y, t) \cdot\left[\vec{e}_{j}+\nabla_{y} \times \vec{w}_{j}(x, y, t)\right] d y \\
& =\int_{y}\left[\vec{e}_{i}+\nabla_{y} \times \vec{w}_{i}(x, y, t)\right] \cdot \mathscr{C}(\widehat{\vec{H}}(x, t), x, y, t) \cdot\left[\vec{e}_{j}+\nabla_{y} \times \vec{w}_{j}(x, y, t)\right] d y
\end{aligned}
$$

for a.e. $(x, t) \in \Omega_{T}(i, j=1,2,3),(7.28)$ is thus established.

The tensor $\mathscr{C}_{0}$ is symmetric whenever so is $\mathscr{C}$, but the off-diagonal elements of $\mathscr{C}_{0}$ need not vanish even if the tensor $\mathscr{C}$ is diagonal; a coarse-scale isotropic medium may indeed be the outcome of the homogenization of a mesoscopically isotropic material. This is easily understandable, for the fine-scale arrangement need not be isotropic.

\section{Discussion and Conclusions}

Methods of Passage to the Limit. In Sec. 5 in the proof of existence of a solution and in Sec. 6 in the derivation of the two-scale homogenized problem, we had to cope with the double nonlinearity of the parabolic-hyperbolic system of P.D.E.s. Because of the monotonicity of the two constitutive relations, in order to pass to the limit in the latter it sufficed to take the limit in the integral of suitable products, and then apply Propositions 4.2 and 4.3. 
In Sec. 5 we used compensated compactness to deal with the limit of the integral $\iint_{\mathbf{R}_{T}^{3}} \vec{B}_{\varepsilon m} \cdot \vec{H}_{\varepsilon m} \theta d x d t$ (that we wrote implying $\varepsilon$ ) as $m \rightarrow \infty$; this provided the relation between $\vec{B}_{\varepsilon}$ and $\vec{H}_{\varepsilon}$. By assuming $\varphi$ to be strictly convex w.r.t. its first argument, we also proved the strong $L^{2}$-convergence of the sequence $\left\{\vec{H}_{\varepsilon m}\right\}$. This allowed us to derive the relation between $\vec{J}_{\varepsilon}$ and the pair $\left(\vec{E}_{\varepsilon}, \vec{H}_{\varepsilon}\right)$, without the need of any further compactness property. This strategy may be compared with that of Visintin (1996, Chap. III), and is at variance with Alt and Luckhaus (1983) and DiBenedetto and Showalter (1981). Further technicalities were needed, because of the occurrence of curls in place of gradients and of the consequent weakness of the a priori estimates.

The estimates that we derived for the solution of Problem $5.1_{\varepsilon}$ allowed us to apply weak two-scale compactness, cf. Proposition 3.1, getting existence of a weakly two-scale convergent subsequence of solutions. We were then able to show that its two-scale limit solves the two-scale homogenized Problem 6.1. There is a strict analogy between the methods that we used to prove existence of a solution of Problem 5.1 and those by which we achieved the two-scale homogenization. The very possibility of extending standard single-scale procedures to two-scale convergence confirms the potentialities of the two-scale notion.

It is clear that the analysis is much simpler whenever either of the two constitutive relations is linear: in that case the problem is reduced to a single variational inequality, for which one can easily prove existence and uniqueness of the solution of Problem 5.1 $1_{\varepsilon}$ without using compactness properties. An analogous simplification occurs in the two-scale homogenization procedure, and in this case one can also show that the $\varepsilon$-problem and its homogenized are well-posed.

Generalizations and Open Questions. As we saw in Sec. 2, the Hall effect corresponds to a dependence of $\vec{J}$ on both $\vec{E}$ and $\vec{B}$ in $\Omega_{T}$, cf. (2.10); this may be represented by a continuous mapping $(\vec{E}, \vec{H}) \mapsto \vec{J}$ only if the (monotone) constitutive relation $\vec{H} \mapsto \vec{B}$ is continuous. However ferromagnetic materials exhibit discontinuities in the dependence of $\vec{B}$ on $\vec{H}$, so that in order to prove existence of a solution of the Maxwell system one should be able to pass to the limit as $m \rightarrow \infty$ in the integral $\int_{\Omega} \vec{J}_{\varepsilon m} \times \vec{B}_{\varepsilon m} d x$. We know that both factors are divergence-free, but this does not allow one to apply compensated compactness. We thus leave the existence of a solution of Problem $5.1_{\varepsilon}$ with a discontinuous relation between $\vec{H}_{\varepsilon}$ and $\vec{B}_{\varepsilon}$ as an open question, let alone for the homogenization of this problem.

The developments of this paper might be extended in several directions. For instance one might deal with functions $\varphi$ and $\vec{\alpha}$ that explicitly depend on time. If the latter were rapidly oscillating in time one might also consider timehomogenization, possibly using a two-scale formulation also in this case. This would be quite appropriate, for a fine time-scale is typically associated to any fine lengthscale. Dealing with the homogenization of a nonperiodic medium would also be of applicative interest.

Open questions include the possible uniqueness of the solution of the problems of Secs. 4-7; the occurrence of two nonlinearities in the principal part makes this issue nontrivial, also considering that this setting does not seem to fit that of the uniqueness results of Blanchard and Porretta (2005), Carrillo (1999), Carrillo and Wittbold (1999), and Igbida and Urbano (2003).

If the medium were an insulator, or in presence of a rapidly varying source-field, displacement currents should not be neglected in $\Omega$; this would yield a quasilinear hyperbolic equation, and in this case even existence of a solution looks challenging. 
Quasi-Stationary Processes. For quasi-stationary evolution the system of Maxwell's equations (2.1)-(2.4) are replaced by the magnetostatic equations

$$
c \nabla \times \vec{H}=4 \pi \vec{J}, \quad \nabla \cdot \vec{B}=0 \quad \text { in } \mathbf{R}_{T}^{3},
$$

coupled with a constitutive relation of the form $\vec{B} \in \partial \varphi(\vec{H}, x, x / \varepsilon)$ in $\mathbf{R}_{T}^{3}$; here $\vec{J}$ is a prescribed field. This problem may be formulated as a single variational inequality; well-posedness can easily be proved, and corresponding two-scale and single-scale homogenized problems can be derived along the lines of Secs. 6, 7; cf. Visintin (in preparation). The same applies to the electrostatic equations

$$
c \nabla \cdot \vec{D}=4 \pi \rho_{\mathrm{el}}, \quad \nabla \times \vec{E}=\overrightarrow{0} \text { in } \mathbf{R}_{T}^{3}
$$

coupled with a constitutive relation of the form $\vec{D} \in \partial \varphi(\vec{E}, x, x / \varepsilon)$ in $\mathbf{R}_{T}^{3}$, for a prescribed electric charge distribution $\rho_{\mathrm{el}}$.

\section{Acknowledgment}

This research was partially supported by the project "Free boundary problems, phase transitions and models of hysteresis" of Italian M.U.R..

\section{References}

Allaire, G. (1992). Homogenization and two-scale convergence. SIAM J. Math. Anal. 23:14821518.

Allaire, G. (2002). Shape Optimization by the Homogenization Method. New York: Springer.

Alt, H. W., Luckhaus, S. (1983). Quasilinear elliptic-parabolic differential equations. Math. Z. 183:311-341.

Arai, T. (1979). On the existence of the solution for $\partial \varphi\left(u^{\prime}(t)\right)+\partial \psi(u(t)) \ni f(t)$. J. Fac. Sci. Univ. Tokyo. Sec. IA Math. 26:75-96.

Arbogast, T., Douglas, J., Hornung, U. (1990). Derivation of the double porosity model of single phase flow via homogenization theory. SIAM J. Math. Anal. 21:823-836.

Ashcroft, N. W., Mermin, N. D. (1976). Solid State Physics. Philadelphia: Holt, Rinehart and Winston.

Attouch, H. (1984). Variational Convergence for Functions and Operators. Boston: Pitman.

Bakhvalov, N., Panasenko, G. (1989). Homogenisation: Averaging Processes in Periodic Media. Dordrecht: Kluwer.

Barbu, V. (1976). Nonlinear Semigroups and Differential Equations in Banach Spaces. Leyden: Noordhoff.

Bensoussan, G., Lions, J. L., Papanicolaou, G. (1978). Asymptotic Analysis for Periodic Structures. Amsterdam: North-Holland.

Birnir, B., Wellander, N. (2006). Homogenized Maxwell's equations; a model for ceramic varistors. Discrete Contin. Dyn. Syst. Ser. B 6:257-272.

Blanchard, D., Francfort, G. (1988). Study of a doubly nonlinear heat equation with no growth assumptions on the parabolic term. SIAM J. Math. Anal. 19:1032-1056.

Blanchard, D., Francfort, G. (1991). A few results on a class of degenerate parabolic equations. Ann. Scuola Norm. Sup. Pisa Cl. Sci. 18:213-249.

Blanchard, D., Porretta, A. (2005). Stefan problems with nonlinear diffusion and convection. J. Differential Equations 210:383-428.

Bossavit, A. (1993). Électromagnétisme en Vue de la Modélisation [Electromagnetism in View of Modelling]. Paris: Springer. 
Bossavit, A., Damlamian, A. (1981). Homogenization of the Stefan problem and application to magnetic composite media. IMA J. Appl. Math. 27:319-334.

Bossavit, A., Verité, J. C. (1981). A Mixed Finite Element Boundary Integral Equation Method to Solve the Three Dimensional Eddy Current Problem. Chicago: COMPUMAG Congress.

Bossavit, A., Griso, G. Miara, B. (2005). Modelling of periodic electromagnetic structures. Bianisotropic materials with memory. J. Math. Pures Appl. 84:819-850.

Bourgeat, A., Luckhaus, S., Mikelic, A. (1996). Convergence of the homogenization process for a double-porosity model of immiscible two-phase flow. SIAM J. Math. Anal. 27:1520-1543.

Braides, A., Defranceschi, A. (1998). Homogenization of Multiple Integrals. Oxford: Oxford University Press.

Brezis, H. (1973). Opérateurs Maximaux Monotones et Semi-Groupes de Contractions dans les Espaces de Hilbert. Amsterdam: North-Holland.

Browder, F. (1970). Existence theorems for nonlinear partial differential equations. In: Chern, S., Smale, S., eds. Proceedings of Symposia in Pure Mathematics, Vol. XVI. Providence: AMS, pp. 1-60.

Carbone, L., Sbordone, C. (1979). Some properties of $\Gamma$-limits of integral functionals. Ann. Mat. Pura Appl. 122:1-60.

Carbone, L., De Arcangelis, R. (2001). Unbounded Functionals in the Calculus of Variations. Representation, Relaxation, and Homogenization. Boca Raton: Chapman and Hall/CRC.

Carrillo, J. (1999). Entropy solutions for nonlinear degenerate problems. Arch. Ration. Mech. Anal. 147:269-361.

Carrillo, J., Wittbold, P. (1999). Uniqueness of renormalized solutions of degenerate ellipticparabolic problems. J. Differential Equations 156:93-121.

Carroll, R. W., Showalter, R. E. (1976). Singular and Degenerate Cauchy Problems. New York: Academic Press.

Cessenat, M. (1996). Mathematical Methods in Electromagnetism. River Edge: World Scientific.

Cherkaev, A., Kohn, R., eds. (1997). Topics in the Mathematical Modelling of Composite Materials. Boston: Birkhäuser.

Cioranescu, D., Donato, P. (1999). An Introduction to Homogenization. New York: Oxford Univ. Press.

Cioranescu, D., Damlamian, A., Griso, G. (2002). Periodic unfolding and homogenization. C.R. Acad. Sci. Paris, Ser. I 335:99-104.

Cioranescu, D., Damlamian, A., De Arcangelis, R. (2004). Homogenization of nonlinear integrals via the periodic unfolding method. C.R. Acad. Sci. Paris, Ser. I 339:77-82.

Cioranescu, D., Damlamian, A., De Arcangelis, R. (2006). Homogenization of quasiconvex integrals via the periodic unfolding method. SIAM J. Math. Anal. 37:1435-1453.

Codegone, M., Negro, A. (1982). Homogenization of a nonlinear quasistationary Maxwell problem. Portugal. Math. 41:277-285.

Codegone, M., Negro, A. (1984). Homogenization of the nonlinear quasistationary Maxwell equations with degenerated coefficients. Appl. Anal. 18:159-173.

Colli, P. (1992). On some doubly nonlinear evolution equations in Banach spaces. Japan J. Indust. Appl. Math. 9:181-203.

Colli, P., Visintin, A. (1990). On a class of doubly nonlinear evolution problems. Comm. in PDEs 15:737-756.

Dal Maso, G. (1993). An Introduction to $\Gamma$-Convergence. Boston: Birkhäuser.

Damlamian, A. (1981). Homogenization for Eddy Currents. Delft Progress Report 6:268-275.

Dautray, R., Lions, J. L. (1988). Analyse Mathématique et Calcul Numérique [Mathematical Analysis and Numerical Calculus]. Vol. 6, Paris: Masson.

De Giorgi, E., Spagnolo, S. (1973). Sulla convergenza degli integrali dell'energia per operatori ellittici del secondo ordine [On the convergence of the energy integrals for elliptic operators of second order]. Boll. Un. Mat. Ital. 8:391-411. 
DiBenedetto, E., Showalter, R. E. (1981). Implicit degenerate evolution equations and applications. SIAM J. Math. Anal. 12:731-751.

Duvaut, G., Lions, J. L. (1972). Les Inéquations en Mécanique et en Physique [The Inequalities in Mechanics and Physics]. Paris: Dunod.

Ekeland, I., Temam, R. (1974). Analyse Convexe et Problèmes Variationnelles [Convex Analysis and Variational Problems.]. Paris: Dunod Gauthier-Villars.

Grange, O., Mignot, F. (1972). Sur la résolution d'une équation et d'une inéquation paraboliques non linéaires [On the solution of nonlinear parabolic equations and inequalities]. J. Functional Analysis 11:77-92.

Hiriart-Urruty, J.-B., Lemarechal, C. (1993). Convex Analysis and Optimization Algorithms. Berlin: Springer.

Hornung, U., ed. (1997). Homogenization and Porous Media. New York: Springer.

Igbida, N., Urbano, J. M. (2003). Uniqueness for nonlinear degenerate problems. Nonlinear Differential Equations Appl. 10:287-307.

Jikov, V. V., Kozlov, S. M., Oleinik, O. A. (1994). Homogenization of Differential Operators and Integral Functionals. Berlin: Springer.

Jones, D. S. (1964). The Theory of Electromagnetism. Oxford: Pergamon Press.

Landau, L., Lifshitz, E. (1960). Electrodynamics of Continuous Media. Oxford: Pergamon Press.

Lenczner, M. (1997). Homogénéisation d'un circuit électrique. C.R. Acad. Sci. Paris, Ser. II 324:537-542.

Lenczner, M., Senouci, G. (1999). Homogenization of electrical networks including voltageto-voltage amplifiers. Math. Models Meth. Appl. Sci. 9:899-932.

Lions, J. L. (1969). Quelques Méthodes de Résolution des Problèmes aux Limites Non Linéaires [Some Methods of Solution of Nonlinear Boundary-Value Problems]. Paris: Dunod.

Lukkassen, D., Nguetseng, G., Wall, P. (2002). Two-scale convergence. Int. J. Pure Appl. Math. 2:35-86.

Marcellini, P. (1978). Periodic solutions and homogenization of nonlinear variational problems. Ann. Mat. Pura Appl. 117:139-152.

Milton, G. W. (2002). The Theory of Composites. Cambridge: Cambridge Univ. Press.

Murat, F. (1978). Compacité par compensation [Compensated compactness]. Ann. Scuola Norm. Sup. Pisa 5:489-507.

Murat, F. (1981). Compacité par compensation: condition nécessaire et suffisante de continuité faible sous une hypothèse de rang constant. Ann. Scuola Norm. Sup. Pisa Cl. Sci. 8:69-102.

Murat, F. (1987). A survey on compensated compactness. In: Contributions to Modern Calculus of Variations (Bologna, 1985). Harlow: Longman Sci. Tech., pp. 145-183.

Murat, F., Tartar, L. (1997). H-convergence. In: Cherkaev, A., Kohn, R., eds. Topics in the Mathematical Modelling of Composite Materials. Boston: Birkhäuser, pp. 21-44.

Nandakumaran, A. K., Rajesh, M. (2001). Homogenization of a nonlinear degenerate parabolic differential equation. Electron. J. Differential Equations 17:19, (electronic).

Negro, A. (1987). Homogenization of the quasistationary Maxwell equations in nonlinear laminated materials. Appl. Anal. 25:197-227.

Nguetseng, G. (1989). A general convergence result for a functional related to the theory of homogenization. SIAM J. Math. Anal. 20:608-623.

Rockafellar, R. T. (1968). Integrals which are convex functionals. Pacific J. Math. 24:525-539.

Rockafellar, R. T. (1969). Convex Analysis. Princeton: Princeton University Press.

Sanchez-Palencia, E. (1980). Non-Homogeneous Media and Vibration Theory. New York: Springer.

Tartar, L. (1977). Course Peccot. Paris: Collège de France. (Unpublished)

Tartar, L. (1978). Une nouvelle méthode de résolution d'équations aux dérivées partielles non linéaires [A new method of solution of nonlinear partial differential equations]. In: Journées d'Analyse Non Linéaire. Berlin: Springer, pp. 228-241. 
Tartar, L. (1979). Compensated compactness and applications to partial differential equations. In: Knops, R. J., ed. Nonlinear Analysis and Mechanics: Heriott-Watt Symposium, Vol. IV. pp. 136-212.

Tartar, L. (2002). Mathematical tools for studying oscillations and concentrations: From Young measures to H-measures and their variants. In: Antonić, N., van Duijn, C. J., Jäger, W., Mikelić, A., eds. Multiscale Problems in Science and Technology. Berlin: Springer, pp. 1-84.

Visintin, A. (1984). Strong convergence results related to strict convexity. Communications in PDEs 9:439-466.

Visintin, A. (1985). Study of the Eddy current problem taking account of Hall's effect. Appl. Anal. 19:217-226.

Visintin, A. (1996). Models of Phase Transitions. Boston: Birkhäuser.

Visintin, A. (2004). Some properties of two-scale convergence. Rend. Acc. Naz. Lincei XV:93-107.

Visintin, A. (2006a). Homogenization of doubly-nonlinear equations. Rend. Lincei Mat. Appl. 17:211-222.

Visintin, A. (2006b). Homogenization of the nonlinear Kelvin-Voigt model of visco-elasticity and of the Prager model of plasticity. Continuum Mech. Thermodyn. 18:223-252.

Visintin, A. (2007a). Two-scale convergence of first-order operators. Z. Anal. Anwendungen 26:133-164.

Visintin, A. (2007b). Two-scale convergence of some integral functionals. Calc. Var. Partial Differential Equations 29:239-265.

Visintin, A. (2007c). Homogenization of a doubly-nonlinear Stefan-type problem. SIAM J. Math. Anal. 39:987-1017.

Visintin, A. Upscaling and downscaling in nonlinear homogenization with applications to magnetism. (In preparation).

Wellander, N. (2002). Homogenization of the Maxwell equations. Case I. Linear theory. Appl. Math. 46:29-51. Case II. Nonlinear conductivity. Appl. Math. 47:255-283.

Wellander, N., Kristensson, G. (2003). Homogenization of the Maxwell equations at fixed frequency. SIAM J. Appl. Math. 64:170-195. 